\title{
A COUPLED THERMOMECHANICAL MODEL FOR THE SOLIDIFICATION OF CAST METALS
}

\author{
DIEGO CELENTANO, SERGIO OLLER and EUGENIO OÑATE \\ International Center for Numerical Methods in Engineering, E. T. S. d'Enginyers de Camins, \\ Canals i Ports, Universitat Politècnica de Catalunya, Gran Capità s/n, Mòdul $\mathrm{Cl}$, \\ 08034 Barcelona, Spain
}

(Received 11 June 1994; in revised form 2 March 1995)

\begin{abstract}
A coupled thermomechanical model to simulate solidification problems in casting is presented. The model is formulated from a phenomenological point of view using a general isotropic thermoelasto-plasticity theory. Generalized phase-change effects accounting for the different thermomechanical behaviour of the solidifying material during its evolution from liquid to solid have been considered. For this purpose, a phase-change function, plastic evolution equations and a temperature-dependent constitutive law have been defined. Full thermomechanical effects as well as variable thermal and mechanical boundary conditions are also taken into account.

Particular details concerning the numerical implementation of the model are given, where special emphasis is devoted to the resulting highly non-linear fully coupled finite element equations.

The behaviour of this formulation is studied first in a simple quenching problem. Finally, a cylindrical casting test problem including phase-change phenomena, temperature-dependent constitutive properties and contact effects is analysed. Numerical results are compared with laboratory measurements.
\end{abstract}

\section{INTRODUCTION}

The prediction of the full thermomechanical behaviour of bodies with thermal and mechanical temperature-dependent material properties is of great practical importance in many engineering situations. In particular, a major issue is the formation of cracks due to induced thermal stress fields in casting processes. Although an analytical investigation into thermal stress development has been attempted by many researchers [see e.g. Cassenti and Annigeri (1989); Malvern (1969); Ziegler (1983)], it has long been recognized that the use of numerical method.s is necessary to analyse complex realistic problems.

For this purpose, different thermomechanical formulations with internal variables governed by rate equations [see e.g. Coleman and Gurtin (1967); Lubliner (1972); Lubliner (1990)] have been proposed and successfully applied for the analysis of coupled elastoplastic problems by many researchers [i.e. see e.g. Argyris et al. (1981); Armero and Simo (1992a,b); Bruhns and Služalec (1989); Kleiber (1991); Simo (1991); Služalec (1988); Smelser and Richmond (1988); Wriggers and Miehe (1992); Wriggers et al. (1989)]. Some of these models have been used to simulate solidification processes [see e.g. Smelser and Richmond (1988), Williams et al. (1990) and the references there listed]. Nevertheless, this problem has many complex aspects that are usually difficult to deal with, such as:

- the equilibrium and energy equations are coupled. Consequently, a robust and efficient numerical strategy is crucial for solving the highly non-linear finite element equations,

- a constitutive model which can represent the liquid, mushy and solid phases of the casting is necessary,

- different kinds of materials are usually involved in solidification processes,

- thermal and mechanical changing boundary conditions must be taken into account. This requires the consideration of a pressure/gap-dependent convection-radiation model and a contact-friction formulation, respectively,

- latent heat effects introduce oscillations in the algorithms,

- an accurate residual stress evaluation has to be performed,

- microstructural effects may be important. 
In this paper, a general phenomenological thermoelasto-plastic model taking into account many of previous aspects is presented in Section 2. Furthermore, a thermomechanical contact model, including a gap/pressure-dependent convection-radiation model and a contact formulation, is also presented. As a first approach to the problem, infinitesimal deformation will be assumed and friction effects will be neglected in this work.

In Section 3, the finite element formulation of the coupled thermomechanical model is derived. Special attention is devoted to "non-standard" terms accounting for phase-change, thermomechanical coupling and contact effects.

Two numerical examples are presented in Section 4. A study of the performance of different solution strategies and the influence of the thermomechanical coupling terms when solving the energy equation has been performed by means of a simple quenching example. Finally, the model is validated in the analysis of a cylindrical casting test problem, for which experimental results are available for comparison.

\section{GOVERNING LOCAL EQUATIONS}

\subsection{General thermomechanical formulation}

(a) Basic definitions. Let some open bounded domains $\Omega_{(i)} \subset \mathbb{R}^{n_{\mathrm{dim}}}\left(1 \leqslant n_{\mathrm{dim}} \leqslant 3\right.$ and $i=1, \ldots, n_{\text {body }}$ ) be the reference (initial) configurations of some $n_{\text {body }}$ continuum thermoelasto-plastic bodies $\mathscr{B}_{(i)}$ (that may thermomechanically interact between themselves) with material coordinates labelled by $X \in \bar{\Omega}_{(i)}$ (all of them measured with respect to the same reference coordinate system), $\Gamma_{(i)}=\partial \Omega_{(i)}$ their smooth boundaries respectively, and $\Upsilon \subset \mathbb{R}^{+}$ be the time interval of analysis $(t \in \Upsilon)$. Further, $\varphi_{(i)}\left({ }^{\circ}, t\right): \mathscr{B}_{(i)} \rightarrow \mathbb{R}^{n_{\text {dim }}}$ denotes the configurations of such bodies at time $t$ and, as usual, $\bar{\Omega}_{(i)}=\Omega_{(i)} \cup \Gamma_{(i)}$. In the infinitesimal displacement/strain con text assumed here, the configurations $\varphi_{(i)}$ at dificent times coincide, respectively, with the reference ones. For sirnplicity in the notation, subscript (i) will be dropped from here onwards unless its use facilitates the description of the formulation.

\section{In phase-chang} $\left(\Omega=\cup \Omega_{p h}\right.$ with $p h$ $\left(p=1, \ldots, n_{p}\right), n_{p h}$ being the number of phases or portions of macroscopical homogeneous

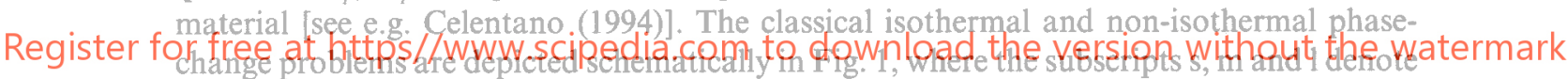
solid, mushy and liquid phases, respectively. Once more, with the purpose of simplifying the notation, indexes $\mathrm{ph}$ and $\mathrm{p}$ will be suppressed in the equations presented below.

A thermomechanical process involving phase-change phenomena can be described by the following local form of the field equations:

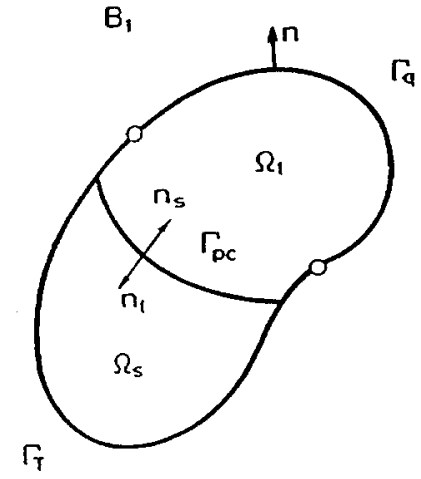

a)

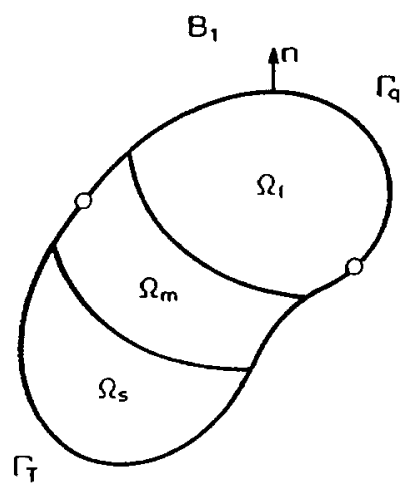

b)

Fig. 1. Geometric description of a non-linear heat conductor $\mathscr{B}_{(1)}$ for phase-change problems. (a) Isothermal case; (b) non-isothermal case. 
- equilibrium condition

$$
\nabla \cdot \sigma+\rho_{0} \mathbf{B}_{F}=\mathbf{0} \text { in } \Omega \times \Upsilon
$$

- First Law of Thermodynamics or balance of energy

$$
\rho_{0} \dot{\omega}=-\nabla \cdot \mathbf{q}+\rho_{0} r+\sigma: \dot{\varepsilon} \quad \text { in } \Omega \times \Upsilon,
$$

- Second Law of Thermodynamics, expressed by the Clausius-Duhem inequality

$$
\rho_{0} \gamma=\rho_{0} \dot{\eta}-\rho_{0} \frac{r}{T}+\nabla \cdot\left[\frac{\mathbf{q}}{T}\right] \geqslant 0 \quad \text { in } \Omega \times \Upsilon \text {, }
$$

\section{together with adequate boundary conditions}
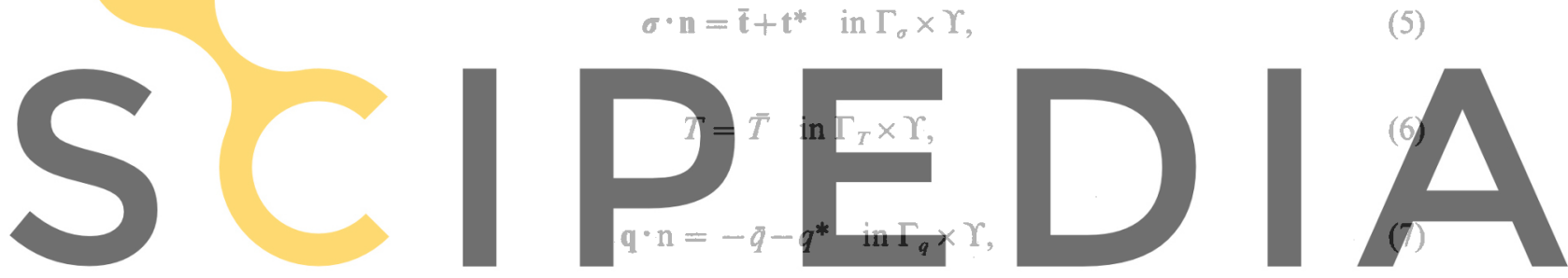

Register foinfreecatdntops//www.scipedia.com to download the version without the watermark

$$
\begin{gathered}
\left.\mathbf{u}(X, t)\right|_{t=0}=\mathbf{u}_{0}(X)=\mathbf{0} \quad \text { in } \Omega, \\
\left.T(X, t)\right|_{t=0}=T_{0}(X) \quad \text { in } \Omega,
\end{gathered}
$$

and appropriate constitutive equations for $\omega, \sigma, \eta$ and $\mathbf{q}$ all defined in $\Omega \times \Upsilon$.

These equations describe a quasi-static coupled thermomechanical initial boundary value problem. Here, $\nabla=\partial / \partial X$ is the gradient operator, $\sigma: \bar{\Omega} \times \Upsilon \rightarrow \mathbb{R}^{n_{\text {dim }}} \times \mathbb{R}^{n_{\text {dim }}}$ is the Cauchy stress second-rank tensor, $\rho_{0}: \Omega \rightarrow \mathbb{R}^{+}$is the density at the reference configuration and $\mathbf{B}_{\mathrm{F}}: \Omega \times \Upsilon \rightarrow \mathbb{R}^{n_{\text {dim }}}$ is the specific body force. Further, $\omega: \Omega \times \Upsilon \rightarrow \mathbb{R}$ is the specific internal energy, $\mathbf{q}: \Omega \times \Upsilon \rightarrow \mathbb{R}^{n_{\text {dim }}}$ is the heat flux vector, $r: \Omega \times \Upsilon \rightarrow \mathbb{R}$ is the specific heat source, $\varepsilon: \Omega \times \Upsilon \rightarrow \mathbb{R}^{n_{\mathrm{dim}}} \times \mathbb{R}^{n_{\mathrm{dim}}}$ is the infinitesimal strain second-rank tensor defined by the usual kinematics relation [see e.g. Malvern (1969)] and $\mathbf{u}: \bar{\Omega} \times \Upsilon \rightarrow \mathbb{R}^{n_{\mathrm{dim}}}$ is the displacement field. The superposed dot implies time derivative while the standard notation for the multiplication of tensors is used [see e.g. Malvern (1969)]. Moreover, in eqn (3) $\gamma: \Omega \times \Upsilon \rightarrow$ $\mathbb{R}$ is the specific internal entropy production rate and $\eta: \Omega \times \Upsilon \rightarrow \mathbb{R}$ is the specific entropy.

In eqns (4) and (5) it is assumed that the displacement field $u(X, t)$ and the stress tensor $\sigma(X, t)$ are prescribed on parts of the boundary $\Gamma_{u} \subset \mathbb{R}^{n_{\mathrm{dim}}-1}$ and $\Gamma_{\sigma} \subset \mathbb{R}^{n_{\mathrm{dim}}-1}$ respectively, where $\mathbf{n}: \partial \Omega \rightarrow \mathbb{R}^{n_{\mathrm{dim}}-1}$ is the unit outward normal to the boundary, $\mathbf{a}: \Gamma_{u} \times \Upsilon \rightarrow \mathbb{R}^{n_{\mathrm{dim}}}$ is the prescribed displacement field, $\overline{\mathbf{t}}: \Gamma_{\sigma} \times \Upsilon \rightarrow \mathbb{P}^{n_{\text {dim }}}$ is the prescribed traction vector and $\mathbf{t}^{*}: \Gamma_{\sigma} \times \Upsilon \rightarrow \mathbb{R}^{n_{\text {dim }}}$ is the contact traction vector due to the fact that the $\mathscr{B}_{(i)}$ bodies may 

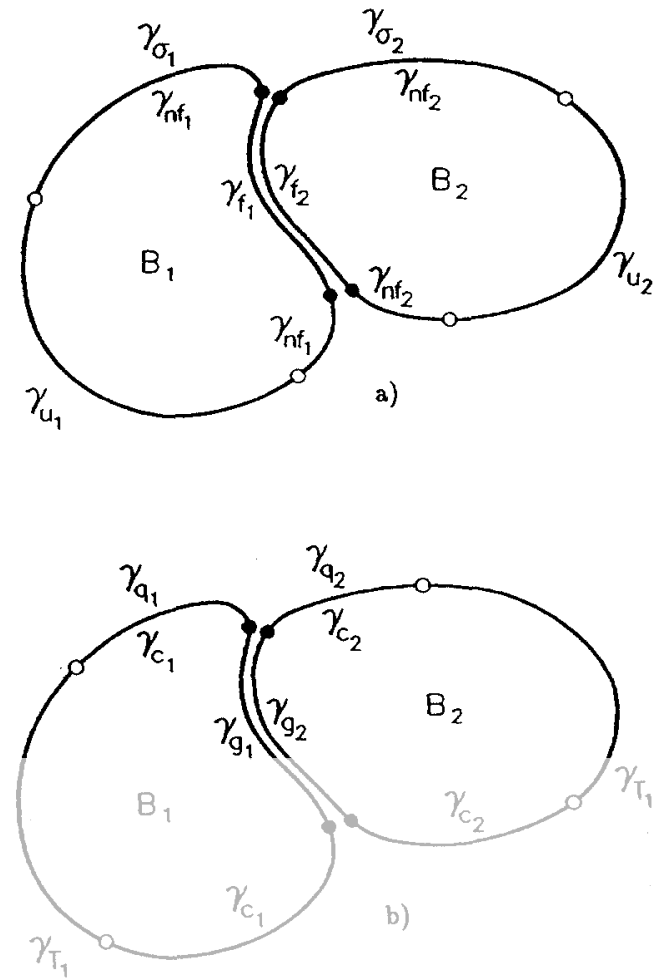

Fig. 2. Geometric description of two non-linear thermoelastic-plastic bodies $\mathscr{O}_{(1)}$ and $\mathscr{B}(2)$ that may thermomechanically interact between themselves. (a) Mechanical boundary conditions; (b) thermal

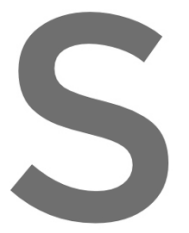
come into contact between
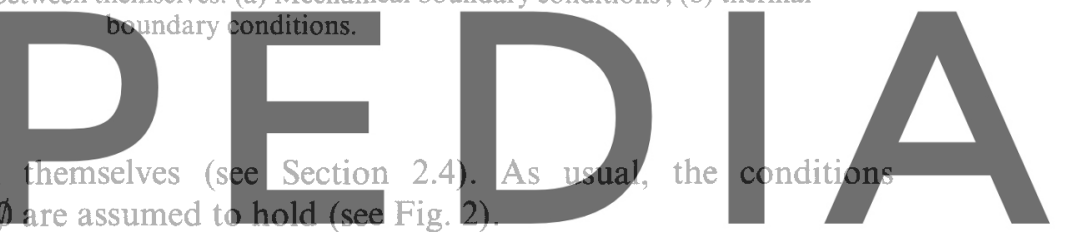

Similarly with eqns (6) and (7), the absolute temperature field $T: \bar{\Omega} \times \Upsilon \rightarrow \mathbb{R}^{+}$and the

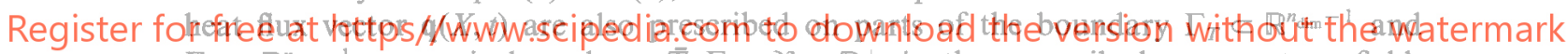
$\Gamma_{q} \in \mathbb{R}^{n_{\mathrm{dim}}-1}$ respectively, where $\bar{T}: \Gamma_{T} \times \Upsilon \rightarrow \mathbb{R}^{+}$is the prescribed temperature field, $\bar{q}: \Gamma_{q} \times \Upsilon \rightarrow \mathbb{R}$ is the prescribed normal heat flux and $q^{*}: \Gamma_{q} \times \Upsilon \rightarrow \mathbb{R}$ is the normal heat flux due to convection-radiation phenomena (see Section 2.3). Once more, the following conditions are considered (see Fig. 2) : $\bar{\Gamma}_{T} \cup \bar{\Gamma}_{q}=\partial \bar{\Omega}$ and $\Gamma_{T} \cap \Gamma_{q}=\emptyset$.

(b) Basic constitutive relations. With the help of the Clausius-Duhem inequality (3) and the definition of the specific Helmholtz free energy $\psi$ [see e.g. Coleman and Gurtin (1967); Malvern (1969); Ziegler (1983)],

$$
\psi=\hat{\psi}\left(\varepsilon, \alpha_{k}, T\right)=\omega-\eta T \text { in } \Omega \times \Upsilon,
$$

the constitutive relations for $\omega, \sigma$ and $\eta$ can be established, where the superposed caret serves to distinguish a state function from its repective value. In eqn (10), $\alpha_{k}: \Omega \times \Upsilon \rightarrow \mathbb{R}^{n}$ int is the $n_{\text {int }}$-dimensional $\left(k=1, \ldots, n_{\text {int }} ; n_{\text {int }} \geqslant 1\right)$ vector field of phenomenological internal state variables governed by rate equations with zero initial values [see e.g. Lubliner (1972); Lubliner (1990)]. Such variables can be scalars or tensors. Moreover, it is important to note that $\varepsilon(X, t), \alpha_{k}(X, t)$ and $T(X, t)$ are assumed to be the independent thermodynamic state variables which determine $\psi$.

The use of the Coleman method [see e.g. Coleman and Gurtin (1967); Lubliner (1972)], taking into account that $\dot{\varepsilon}$ and $\dot{T}$ can be specified arbitrarily in a given thermodynamic state, i.e. if there are no internal constraints, leads to the following relations :

$$
\boldsymbol{\sigma}=\hat{\boldsymbol{\sigma}}\left(\boldsymbol{\varepsilon}, \boldsymbol{\alpha}_{k}, T\right)=\rho_{0} \frac{\partial \psi}{\partial \boldsymbol{\varepsilon}}
$$




$$
\eta=\hat{\eta}\left(\varepsilon, \alpha_{k}, T\right)=-\frac{\partial \psi}{\partial T}
$$

Equation (11) is usually known as the isothermal stress-strain relation [see e.g. Malvern (1969)]. Lubliner (1972) has shown that, in order to establish unique constitutive equations, Coleman's relations (11) and (12) follow even for the definition of $\dot{\boldsymbol{\alpha}}_{k}$ given by the plasticity theory (see Section 2.2).

From eqns (10) and (12), the specific internal energy can be expressed in terms of $\psi$ as:

$$
\omega=\psi-\frac{\partial \psi}{\partial T} T
$$

Finally, the well-known Fourier law is adopted for $\mathbf{q}$ :

$$
\mathbf{q}=-\mathbf{k} \cdot \nabla T
$$

where $\mathbf{k}$ is the conductivity second-rank tensor. This path independent way of defining $\mathbf{q}$ is equivalent to that used by Simo (1991) in terms of a smooth convex heat flux potential function.

(c) Derived constitutive relations. Other important thermodynamic expressions related

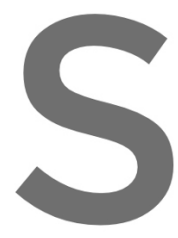

\section{to the behaviour of}
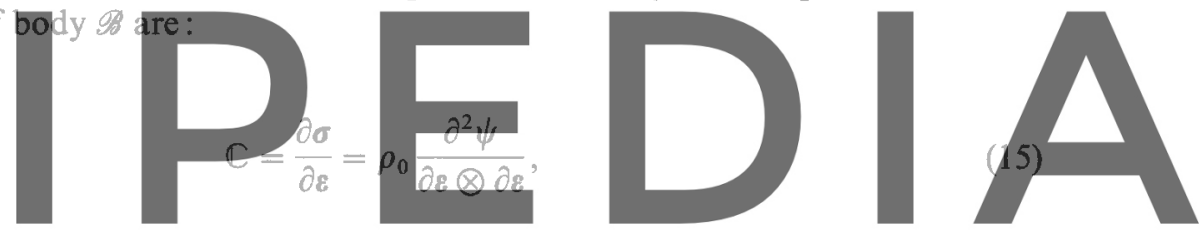

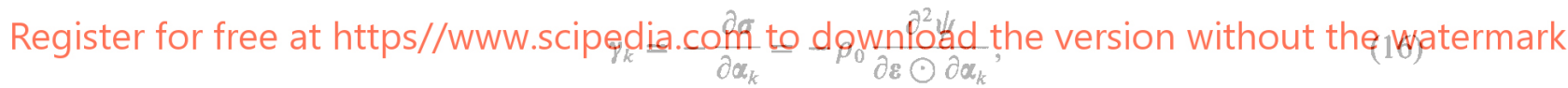

$$
\boldsymbol{\beta}=-\frac{\partial \boldsymbol{\sigma}}{\partial T}=-\rho_{0} \frac{\partial^{2} \psi}{\partial \boldsymbol{\varepsilon} \partial T}
$$

where $\mathbb{C}$ is the elastic tangent constitutive fourth-rank tensor (isothermal) [see e.g. Malvern (1969)], $\gamma_{k}$ are the plastic tangent constitutive variables (once more, they can be scalars or tensors) [see Celentano (1994); Lubliner (1972)] and $\boldsymbol{\beta}$ is the tangent conjugate of the thermal dilatation second-rank tensor [see e.g. Lubliner (1990)]. The symbol $\odot$ indicates the appropriate multiplication according with the nature of each internal variable $\boldsymbol{\alpha}_{k}$.

In phase-change problems, the following general definition is adopted [see e.g. Celentano (1994); Celentano et al. (1993)] :

$$
c=-T \frac{\partial^{2} \psi}{\partial T^{2}}-L \frac{\partial f_{\mathrm{pc}}(T)}{\partial T}
$$

where $c: \Omega \times \Upsilon \rightarrow \mathbb{R}^{+}$is the specific heat capacity, $L$ is the specific latent heat (released in a freezing problem or absorbed in a melting one) and $f_{\mathrm{pc}}$ is the "phase-change" function [see e.g. Celentano (1994); Celentano et al. (1993)]. In an isothermal phase-change problem $f_{\mathrm{pc}}=H\left(T-\bar{T}_{\mathrm{m}}\right)$, with $H$ being the Heaviside function and $\bar{T}_{\mathrm{m}}$ the melting temperature (see 
Fig. 3). On the other hand, when the phase-change occurs in the range of temperatures $\left(T_{1}-T_{\mathrm{s}}\right), f_{\mathrm{pc}}=f$ with:

$$
f(T)= \begin{cases}0 & ; \forall T \leqslant T_{\mathrm{s}} \\ 0<g(T) \leqslant 1 & ; T_{\mathrm{s}}<\forall T \leqslant T_{1} \\ 1 & ; \forall T>T_{1} .\end{cases}
$$

where $T_{1}$ and $T_{\mathrm{s}}$ are the liquidus and solidus temperatures, respectively [see e.g. Celentano et al. (1994)]. Function $g(T)$ may be obtained using a microstructure model [see e.g. Thévoz et al. (1989) and references therein]. However, from a macroscopical point of view assumed in this paper, the simplest choice for $g(T)$ is the linear one (see Fig. 3) $: g(T)=\left(T-T_{5}\right) /$ $\left(T_{1}-T_{\mathrm{s}}\right) \forall T_{\mathrm{s}}<T \leqslant T_{1}$. It should be noted that the temperature derivative of the phasechange function does not exist for certain temperatures. Nevertheless, as it will be shown later, there is no need to evaluate such derivatives in the energy equation (20) [see e.g. Celentano (1994); Celentano et al. (1994)].

A further generalization of eqn (18) takes place when two or more phase-changes occur, i.e. $n_{\mathrm{pc}} \geqslant 2$ [see e.g. Celentano (1994); Celentano et al. (1994)]. In this case, the term $L \partial f_{\mathrm{pc}} / \partial T$ must be replaced by $\Sigma_{j=1}^{n_{\mathrm{pc}}} L_{j} \partial f_{\mathrm{pc}} / \partial T$, where $L_{j}$ and $f_{\mathrm{pc}}$ are the latent heat and phase-change function associated with the $j$ th phase-change. To facilitate the notation, the simpler form of eqn (18) (i.e. $n_{\mathrm{pc}}=1$ ) is retained [see e.g. Celentano (1994); Celentano et al. (1994)].

(d) Energy equation and coupling terms. Under these considerations, the energy equation can be rewritten as
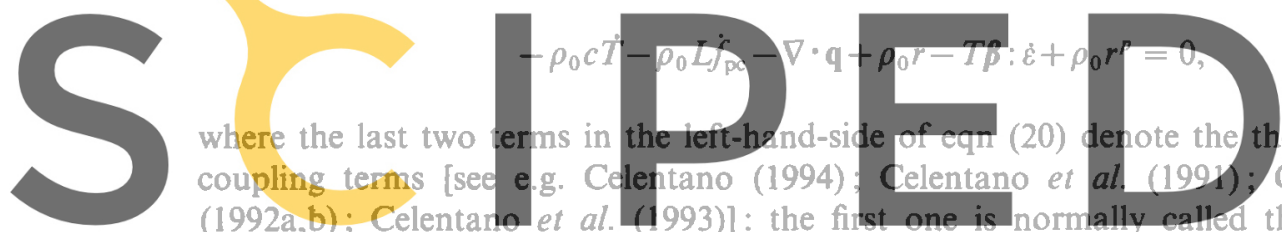

where the last two

coupling terms [see
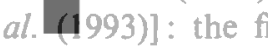

coupling term [see e.g. Ziegler (1983)] while the second accounts for the thermoplastic

$$
r^{p}=\left(T \frac{\partial^{2} \psi}{\partial T \partial \alpha_{k}}-\frac{\partial \psi}{\partial \alpha_{k}}\right) \odot \dot{\alpha}_{k}
$$

(e) Dissipative inequalities. If it is assumed that $\psi$ and $\alpha_{k}$ are independent of $\nabla T$, and $q$ independent of $\alpha_{k}$, the following inequalities, both derived from equation (3), have to be fulfilled [see e.g. Lubliner (1972); Malvern (1969); Ziegler (1983)] :

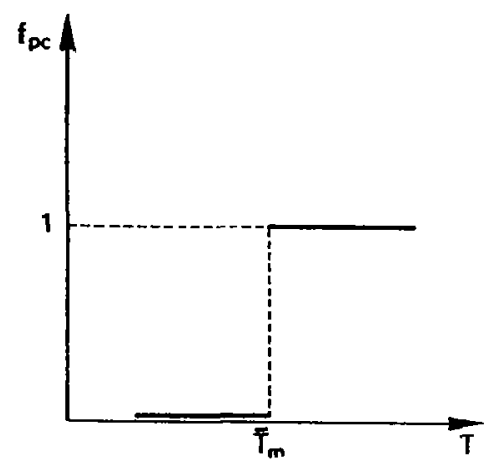

a)

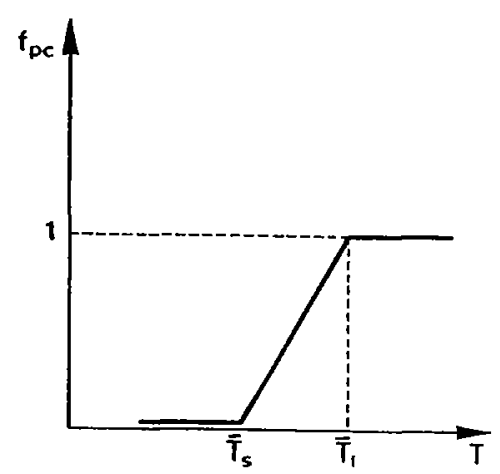

b)

Fig. 3. Phase-change function for (a) isothermal case; (b) non-isothermal case. 


$$
\begin{gathered}
D^{\mathrm{p}}=\rho_{0} \frac{\partial \psi}{\partial \boldsymbol{a}_{k}} \odot \dot{\boldsymbol{\alpha}}_{k} \leqslant 0, \\
D^{\mathrm{th}}=\frac{1}{T} \mathbf{q} \cdot \nabla T \leqslant 0,
\end{gathered}
$$

where $D^{\mathrm{p}}$ and $D^{\text {th }}$ are defined as the plastic and thermal dissipations, respectively. Lubliner (1972) has also shown that eqns (22) and (23) become the necessary and sufficient conditions for the fulfilment of the Clausius-Duhem inequality (3). From eqn (22), it is clear that $\mathbf{q}_{k}=-\partial \psi / \alpha_{k}$ are the conjugate plastic variables of $\boldsymbol{\alpha}_{k}$.

\subsection{Thermoelasto-plastic model}

(a) Basic definitions. In the context of rate-independent plasticity theory, the thermoplastic behaviour of the continuum is governed by a state yield function $F\left(q_{k}\right): \mathbb{R}^{n_{\text {int }}} \rightarrow \mathbb{R}$ (assumed strictly convex and, for simplicity, defined in terms of a unique smooth function), such that no plastic evolutions occur when $F<0$. In accordance with the work of Celentano (1994), a particular model takes place when the yield function is assumed to be of the form $F=\hat{F}\left(\sigma, \mathscr{C}^{\mathrm{P}}, T\right)$, where $\mathscr{C}^{\mathrm{P}}: \Omega \times \Upsilon \rightarrow \mathbb{R}$ is the plastic hardening function [see e.g. Lubliner (1972)]. Now, the thermoelastic admissible domain (also assumed convex) $\mathbb{E}^{\text {te }}$ is defined as [see e.g. Simo (1991)] :

$$
\mathbb{E}^{\text {te: }}=\left\{\left(\sigma, \mathscr{C}^{\mathrm{p}}, T\right) \in\left(\mathbb{R}^{n_{\mathrm{dim}}} \times \mathbb{R}^{n_{\mathrm{dim}}}\right) \times \mathbb{R} \times \mathbb{R}^{+} \mid F\left(\sigma, \mathscr{C}^{\mathrm{p}}, T\right)<0\right\},
$$

and the thermoplastic domain is:
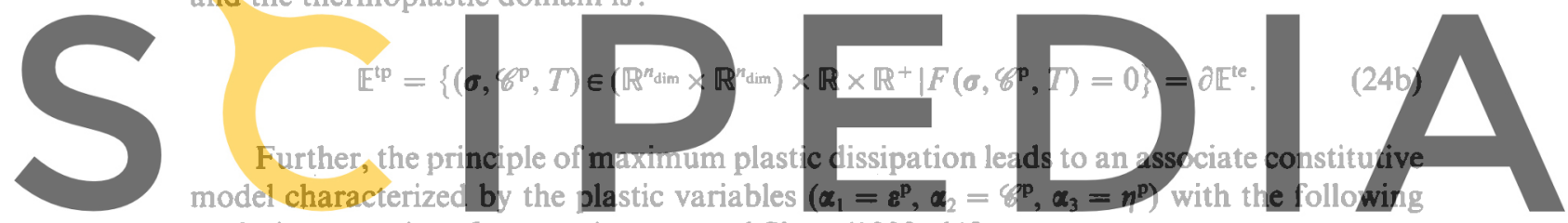

evolution equations [see e.g. Armero and Simo (1992a,b)]:

Register for free at https//www.scipedia.com to download the version without the watermark

$$
\begin{gathered}
\dot{\mathscr{E}}^{\mathrm{p}}=\frac{\partial F}{\partial \sigma} \lambda, \\
\dot{\mathscr{C}}^{\mathrm{p}}=\boldsymbol{H}_{\mathscr{\boldsymbol { q }} \mathrm{p}} \dot{\lambda} \\
\dot{\eta}^{\mathrm{p}}=\frac{1}{\rho_{\mathbf{0}}} \frac{\partial F}{\partial \boldsymbol{T}} \dot{\lambda},
\end{gathered}
$$

together with the load-unload Kuhn-Tucker conditions [see e.g. Armero and Simo (1992a,b) ; Simo (1991)] $\dot{\lambda} \geqslant 0, F \leqslant 0, \dot{\lambda} F=0$ and the consistency condition [see e.g. Armero and Simo (1992a,b); Simo (1991)] $\dot{\lambda} \dot{F}=0$, where $\varepsilon^{p}: \Omega \times \Upsilon \rightarrow \mathbb{R}^{n_{\text {dim }}} \times \mathbb{R}^{n_{\text {dim }}}$ is the plastic strain second-rank tensor, $\eta^{\mathrm{p}}: \Omega \times \Upsilon \rightarrow \mathbb{R}$ is the plastic entropy and $H_{\mathscr{q}}(T): \Omega \times \Upsilon \rightarrow \mathbb{R}$ is the plastic hardening modulus given by:

$$
H_{\mathscr{Q P}}=h_{\mathscr{P}}(T) \boldsymbol{\sigma}: \mathbf{R}
$$

where $h_{\mathrm{cp}}(T): \Omega \times \Upsilon \rightarrow \mathbb{R}$ is the plastic hardening coefficient and $\mathbf{R}=\partial F / \partial \boldsymbol{\sigma}$ is the flow potential. It can be seen from eqns (26) and (28) that $\dot{\mathscr{C}}^{\mathrm{p}}$ is defined in terms of $\dot{\varepsilon}^{\mathrm{p}}$ [see e.g. Celentano et al. (1991); Celentano et al. (1992a,b); Celentano et al. (1993); Celentano (1994)].

(b) Proposed specific free energy function. Restricting the analysis to the thermoelastoplastic isotropic response, the free energy $\psi$ is formulated as [see e.g. Armero and Simo 
(1992a,b); Celentano (1994); Celentano et al. (1991); Celentano et al. (1992a,b); Celentano et al. (1993)]:

$$
\psi=\hat{\psi}_{\mathrm{te}}\left(\varepsilon-\varepsilon^{\mathrm{p}}, T\right)+\hat{\psi}_{\mathrm{p}}\left(\mathscr{C}^{\mathrm{p}}, \eta^{\mathrm{p}}, T\right)+\hat{\psi}_{\mathrm{pc}}(T),
$$

where $\psi_{\mathrm{te}}, \psi_{\mathrm{tp}}$ and $\psi_{\mathrm{pc}}$ are the thermoelastic, thermoplastic and phase-change parts of $\psi$, respectively. It should be noted that eqn (29) is a partially decoupled form of the specific free energy $\psi$. Choosing the initial temperature as the reference one [see e.g. Celentano (1994)], the thermoelastic part $\psi_{\mathrm{te}}$ is written as :

$$
\begin{aligned}
\psi_{\mathrm{te}}=\hat{\psi}_{\mathrm{te}}\left(\varepsilon-\varepsilon^{\mathrm{p}}, T\right)=\frac{1}{2 \rho_{0}}\left(\varepsilon-\varepsilon^{\mathrm{p}}\right): \mathbb{C}^{\mathrm{s}}:\left(\varepsilon-\varepsilon^{\mathrm{p}}\right)-\frac{1}{\rho_{0}} \beta^{s}:\left(\varepsilon-\varepsilon^{\mathrm{p}}\right)\left(T-T_{0}\right) \\
\quad+\psi_{0}-\eta_{0}\left(T-T_{0}\right)+\frac{1}{\rho_{0}} \sigma_{0}:\left(\varepsilon-\varepsilon^{\mathrm{p}}\right)+c^{\mathrm{s}}\left(T-T_{0}\right)-c^{\mathrm{s}} T \ln \frac{T}{T_{0}},
\end{aligned}
$$

the thermoplastic part is:

$$
\psi_{\mathrm{tp}}=\hat{\psi}_{\mathrm{tp}}\left(\mathscr{C}^{\mathrm{p}}, \eta^{\mathrm{p}}, T\right)=-\frac{\mathscr{C}^{\mathrm{p}}}{\rho_{0}}-T \eta^{\mathrm{p}}
$$

and the phase-change part is:
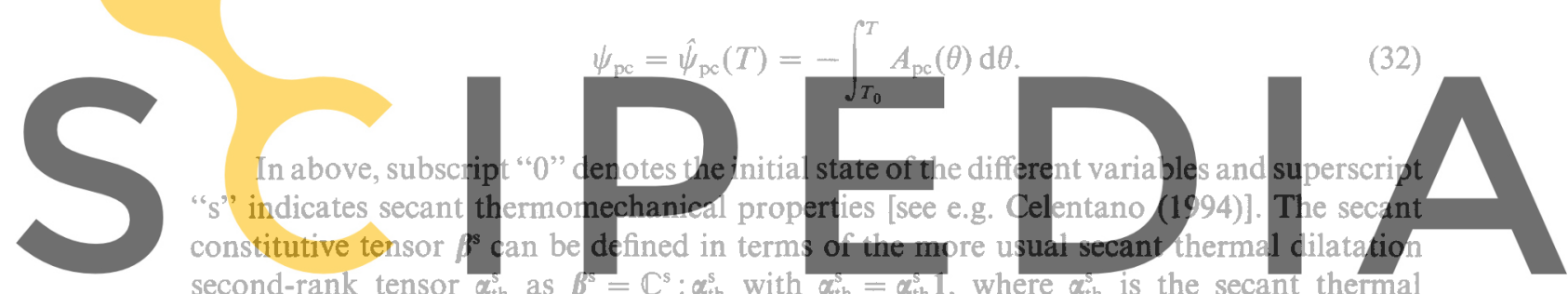

dilatation coefficient [see e.g. Malvern (1969); Ziegler (1983)]. A proposed constitutive

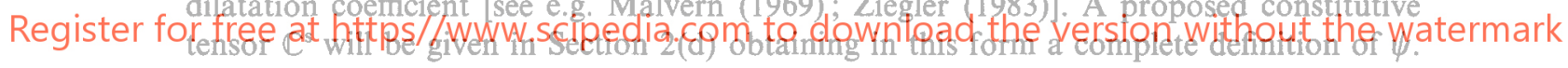

Furthermore, $A_{\mathrm{pc}}$ is defined as [see e.g. Celentano (1994)]:

$$
A_{\mathrm{pc}}(T)=\int_{T_{0}}^{T} \frac{L}{\theta} \frac{\partial f_{\mathrm{pc}}(\theta)}{\partial \theta} \mathrm{d} \theta
$$

where $\theta$ is a dummy variable. The thermoelastic part $\psi_{\mathrm{te}}$ expressed by eqn (30) is a generalization of the usual specific free energy definition given in the thermoelastic context [see e.g. Malvern (1969); Ziegler (1983)]. On the other hand, the thermoplastic part $\psi_{\text {tp }}$ takes into account the irreversibility of the thermomechanical process [see e.g. Celentano (1994)]. Moreover, the phase-change part $\psi_{\mathrm{pc}}$ includes generalized phase-change effects by means of the phase-change function $f_{\mathrm{pc}}$ [see e.g. Celentano (1994)].

This definition of $\psi$ constitutes a crucial point of the model since it is the basis for deriving all the constitutive equations presented in Section 2.1 [see e.g. Celentano (1994)]. In particular, the secant and tangent constitutive laws, the specific heat capacity and the thermoplastic coupling term will be shown below.

(c) Secant and tangent constitutive laws. The thermoelasto-plastic secant constitutive law is obtained using eqn (11) as :

$$
\sigma=\hat{\sigma}\left(\varepsilon-\varepsilon^{\mathrm{p}}, T\right)=\mathbb{C}^{\mathrm{s}}:\left(\varepsilon-\varepsilon^{\mathrm{p}}\right)-\boldsymbol{\beta}^{\mathrm{s}}\left(T-T_{0}\right)+\sigma_{0}
$$

It should be noted that this secant or hyperelastic constitutive law circumvents the 
usual thermodynamic constraints [see e.g. Cassenti and Annigeri (1989); Coleman and Gurtin (1967)] and it depends exclusively on the thermoelastic part of the free energy function.

Assuming that $\mathbb{C}^{s}$ and $\boldsymbol{\beta}^{\text {s }}$ may be temperature-dependent tensors, and using standard plasticity theory [see e.g. Lubliner (1990)], the tangent constitutive law can be written as [see e.g. Celentano (1994)] :

$$
\dot{\boldsymbol{\sigma}}=\mathbb{C}^{\mathrm{ep}}: \dot{\boldsymbol{\varepsilon}}-\boldsymbol{\beta}^{\mathrm{ep}} \dot{T}
$$

where the elasto-plastic tangent constitutive fourth-rank tensor $\mathbb{C}^{\mathrm{ep}}$ is:

$$
\mathbb{C}^{\mathrm{ep}}=\mathbb{C}-\frac{H(\dot{\lambda})}{A} \mathbb{C}: \mathbf{R} \otimes \mathbf{R}: \mathbb{C}
$$

the thermoelasto-plastic tangent constitutive second-rank tensor $\boldsymbol{\beta}^{\text {ep }}$ is :

$$
\boldsymbol{\beta}^{\text {ep }}=\boldsymbol{\beta}-\frac{H(\dot{\lambda})}{A}\left(\mathbf{R}: \boldsymbol{\beta}-\frac{\partial F}{\partial T}\right) \boldsymbol{C}: \mathbf{R},
$$

\section{the plastic consistency parameter is given by:}

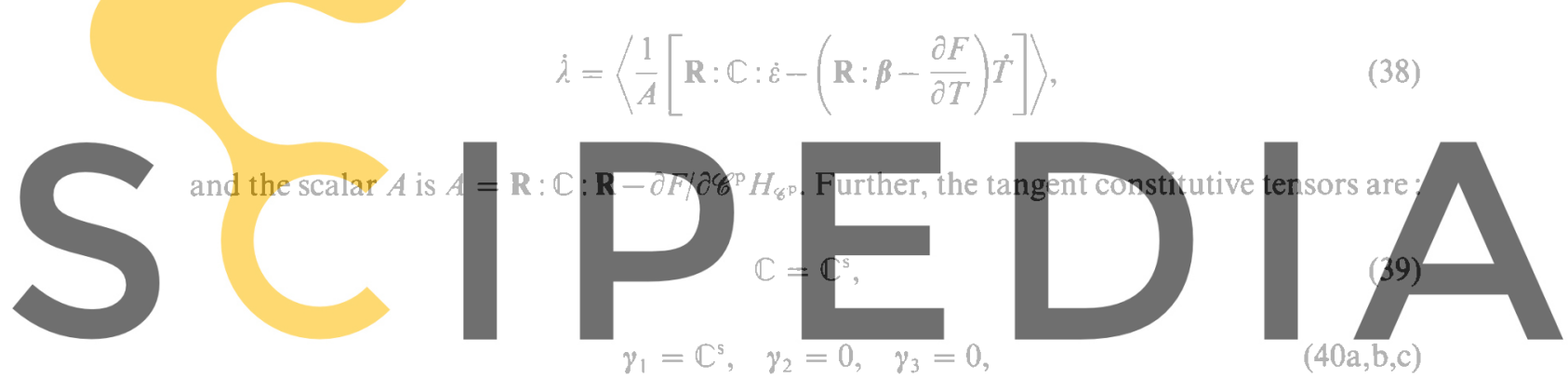

Register for free at https//www.scipedia.com to download the version without the watermark

$$
\beta=\beta^{s}-\frac{\partial C^{s}}{\partial T}:\left(\varepsilon-\varepsilon^{\mathrm{p}}\right)+\frac{\partial \beta^{s}}{\partial T}\left(T-T_{0}\right) \text {. }
$$

With these last considerations, the plastic evolution equations (25)-(27) are completely defined [see e.g. Celentano (1994)].

Equivalent forms of the secant and tangent constitutive laws can be obtained if $\boldsymbol{\beta}^{\mathrm{s}}$ is written in terms of $\boldsymbol{\alpha}_{\mathrm{ch}}^{\mathrm{s}}$ recovering in this case the usual additive decomposition of strains [see e.g. Celentano (1994); Celentano et al. (1991); Celentano et al. (1992a,b); Celentano et al. (1993)].

(d) Proposed constitutive tensor. During solidification, the material in liquid state becomes solid. This means that a qualitative change in its thermomechanical properties is produced. Therefore, this fact should be taken into account in the constitutive tensor written as [see e.g. Celentano (1994); Celentano et al. (1993)]:

$$
\mathbb{C}^{\mathrm{s}}=\mathbb{C}_{\mathrm{vol}}^{\mathrm{s}}+\left(1-f_{\mathrm{pc}}^{\mathrm{ls}}\right) \mathbb{C}_{\mathrm{dev}}^{\mathrm{s}},
$$

where $\mathbb{C}_{\text {vol }}^{\mathrm{s}}$ and $\mathbb{C}_{\text {slev }}^{\mathrm{s}}$ are the volumetric and deviatoric parts of $\mathbb{C}^{\mathrm{s}}$, respectively [see e.g. Malvern (1969); Ziegler (1983)]. Now, $f_{\mathrm{pc}}^{\mathrm{ls}}$ denotes the phase-change function associated with a liquid-solid phase-change [see e.g. Celentano (1994)]. In this case, $f_{\mathrm{pc}}^{\mathrm{ls}}$ and $1-f_{\mathrm{pc}}^{\mathrm{ls}}$ represent the liquid and solid volume fractions, respectively. It should be noted that $f_{\mathrm{pc}}^{\mathrm{ls}}$ has to be a smooth function of $T$ in each phase in order to be able to evaluate the temperature derivatives of $\mathbb{C}^{s}$ needed in the constitutive relations presented in Section 2.1. 


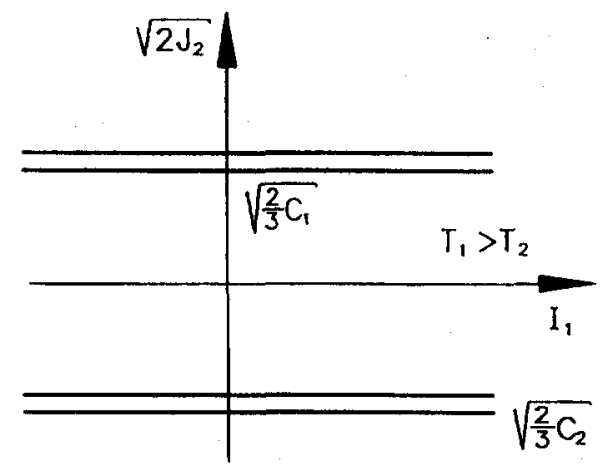

Fig. 4. Von Mises temperature-dependent yield function ( $I_{1}$ is the trace of $\left.\sigma\right)$.

Note that eqn (42) is an additional constitutive assumption implicitly contained in the proposed specific free energy given by eqn (30). With this definition of $\mathbb{C}$, both the classic constitutive law of a liquid in rest (i.e. $\sigma$ is equal to the hydrostatic stress tensor leading to $\sigma^{\prime}=0$ where $\sigma^{\prime}$ is the deviatoric stress tensor) and that corresponding to a solid material can be represented [see e.g. Celentano (1994); Celentano et al. (1993)].

(e) Yield function. A Von Mises temperature-dependent yield function (see Fig. 4) has been adopted as [see e.g. Armero and Simo (1992a,b); Celentano (1994); Wriggers and Miehe (1992); Wriggers et al. (1989)]:
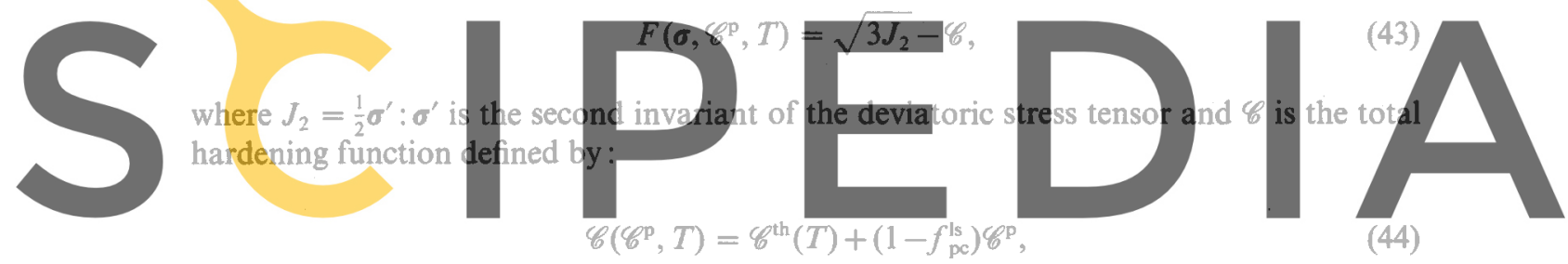

Register for free at https//www.scipedia.com to download the version without the watermark where $\mathscr{G}^{\text {th }}(T)$ is the thermal hardening function (also assumed to be a positive smooth.

function of $T$ ) related to the uniaxial cohesion of the material [see e.g. Celentano (1994)].

Moreover, the flow potential is now given by $R=\left(\sqrt{3} / 2 \sqrt{J_{2}}\right) \sigma^{\prime}$, where it should be noted that $\mathbf{R}$ is non-determinate when $\sigma^{\prime}=\mathbf{0}$. However, as $\mathscr{C}^{\text {th }}>0$, it can be observed that $F<0$ for this particular situation. Therefore, a purely elastic material behaviour $(\dot{\lambda}=0)$ is considered for this case.

With this definition of $F$, the well-known plastic restriction [see e.g. Lubliner (1972); Lubliner (1990)] $\partial F / \partial \boldsymbol{\alpha}_{k} \odot \mathbf{g}_{k}<0$ for $F=\dot{F}=0$ reads $\mathbf{R}: \mathbb{C}: \mathbf{R}+\left(1-f_{\mathrm{pc}}^{\mathrm{ls}}\right) H_{\mathscr{q} \mathrm{p}}>0$ for $F=\dot{F}=0$ which is clearly satisfied if the additional constraint $H_{\mathscr{G}^{\mathrm{p}}}>-\mathbf{R}: \mathscr{C}: \mathbf{R}$ is assumed [see e.g. Lubliner (1972); Lubliner (1990); Celentano (1994)], where the condition $\mathbf{R}: \mathbb{C}: \mathbf{R}>0$ is verified taking into account the definitions given above [see e.g. Celentano (1994)]. In the solid phase $\left(f_{\mathrm{pc}}^{\mathrm{ls}}=0\right)$, the particular situation with $H_{\mathscr{C p}}>0$ is characteristic of work hardening plastic materials [see e.g. Lubliner (1972); Lubliner (1990)].

(f) Specific heat capacity. Taking into account eqn (18) (considering, once more, the definition of the specific free energy given above), the specific heat capacity is expressed as [see e.g. Celentano (1994)] :

$$
\begin{aligned}
c=\hat{c}\left(\varepsilon-\varepsilon^{\mathrm{p}}, T\right)= & -\frac{T}{2 \rho_{0}}\left(\varepsilon-\varepsilon^{\mathrm{p}}\right): \frac{\partial^{2} \mathbb{C}^{\mathrm{s}}}{\partial T^{2}}:\left(\varepsilon-\varepsilon^{\mathrm{p}}\right)+\frac{T}{\rho_{0}} \frac{\partial^{2} \boldsymbol{\beta}^{\mathrm{s}}}{\partial T^{2}}:\left(\varepsilon-\varepsilon^{\mathrm{p}}\right)\left(T-T_{0}\right) \\
& +\frac{2 T}{\rho_{0}} \frac{\partial \boldsymbol{\beta}^{\mathrm{s}}}{\partial T}:\left(\varepsilon-\varepsilon^{\mathrm{p}}\right)+c^{\mathrm{s}}-T \frac{\partial^{2} c^{\mathrm{s}}}{\partial T^{2}}\left[\left(T-T_{0}\right)-T \ln \frac{T}{T_{0}}\right]+2 T \frac{\partial c^{\mathrm{s}}}{\partial T} \ln \frac{T}{T_{0}} .
\end{aligned}
$$


It can be clearly observed that eqn (18) preserves the classical definition of the specific heat capacity in the whole domain because the phase-change part of $\psi$ does not play any role in the expression of $c$. In the particular case of absence of temperature-dependent properties, $c=c^{s}$ is obtained [see e.g. Celentano (1994)].

Further, considering that $\varepsilon-\varepsilon^{\mathrm{p}}$ is the thermoelastic deformation term (in the free energy definition sense), it should be noted that the specific heat capacity does not depend directly on plastic variables. This assumption is typical of materials with uncoupled instantaneous elasticity and it is also usually accepted for metals [see e.g. Lubliner (1972); Lubliner (1990)].

(g) Thermoplastic coupling term. The thermoplastic coupling term defined in eqn (21) is obtained as :

$$
r^{\mathrm{p}}=r_{\varepsilon}^{\mathrm{p}}: \dot{\varepsilon}+r_{T}^{\mathrm{p}} \dot{T}
$$

where

$$
\begin{aligned}
& r_{\varepsilon}^{\mathrm{p}}=H(\dot{\lambda})\left[(\sigma+T \beta): \mathbf{R}+H_{\mathscr{\sigma}}\right] \Phi, \\
& r_{T}^{\mathrm{p}}=H(\dot{\lambda})\left[(\sigma+T \beta): \mathbf{R}+H_{\mathscr{\sigma}}\right] \chi,
\end{aligned}
$$

with $\Phi=R: \mathbb{C} / A$ and $\chi=-(\mathbf{R}: \beta-\partial F / \partial T) / A$.

(h) Constitutive inequalities. Assuming isotropic heat conduction response, the conductivity tensor is written as $\mathbf{k}=k 1$, where $k: \Omega \times \Upsilon \rightarrow \mathbb{R}^{+}$is the conductivity coefficient. Therefore, it should be noted that the thermal dissipation equation (23) is clearly fulfilled if $k \geqslant 0$. Moreover, the plastic dissipation equation (22) becomes now:

$$
\rho_{0}\left[\frac{\partial \psi}{\partial \epsilon^{\mathrm{p}}}: \mathbf{R}+\frac{\partial \psi}{\partial \mathscr{C}^{\mathrm{p}}} H_{\mathscr{G}^{\mathrm{p}}}+\frac{\partial \psi}{\partial \eta^{\mathrm{p}}} \frac{\partial F}{\partial T}\right] \dot{\hat{\lambda}}=\left[-\frac{\sqrt{3}}{2 \sqrt{J_{2}}} \sigma: \sigma^{\prime}-H_{\mathscr{F}^{\mathrm{p}}}+\rho_{0} T\left(\frac{\partial \mathscr{C}^{\mathrm{th}}}{\partial T}-\frac{\partial f_{\mathrm{pc}}^{\mathrm{ls}}}{\partial T} \mathscr{C}^{\mathrm{p}}\right)\right] \dot{\lambda} \leqslant 0 .
$$

This condition is automatically satisfied if there is no evolution of the internal variables $(\dot{\lambda}=0)$. If this is not the case, it is clear that the first term in the left-hand-side of eqn (48) is less than zero and, in order to guarantee the fulfilment of such equations, the following three sufficient conditions are assumed : (i) $H_{\mathscr{S}} \geqslant 0$, (ii) $\partial \mathscr{C}^{\mathrm{th}} / \partial T \leqslant 0$ and (iii) $\partial f_{\mathrm{pc}}^{\mathrm{ls}} / \partial T \geqslant 0$. The first condition is related to the hardening behaviour of the material and guarantees that $\mathscr{C}^{\mathrm{p}} \geqslant 0$, while the second one refers to the thermal softening effect [see e.g. Armero and Simo $(1992 \mathrm{a}, \mathrm{b})]$. Finally, the third condition is satisfied taking into account the definition of $f_{\mathrm{pc}}^{\mathrm{s}}$ given in Section 2.1.

\subsection{Thermal contact model}

(a) Basic definitions. The following conditions are assumed to hold $\Gamma_{c} \cup \Gamma_{g}=\Gamma_{q}$ and $\Gamma_{\mathrm{c}} \cap \Gamma_{\mathrm{g}}=\emptyset$, where $\Gamma_{\mathrm{c}} \subset \mathbb{R}^{n_{\mathrm{dim}}-1}$ and $\Gamma_{\mathrm{g}} \subset \mathbb{R}^{n_{\mathrm{dim}}-1}$ are parts of $\Gamma_{q}$ where the convectionradiation phenomenon takes place between body $\mathscr{B}$ and : (a) the environment around it, and (b) another body due to the gap existing among them, respectively (see Fig. 2). The heat fluxes corresponding to these two cases are denoted by $q_{\mathrm{conv}}$ and $q_{\mathrm{g}}$, respectively. Therefore, the normal heat flux to be considered in the boundary equation (7) is :

$$
q^{*}= \begin{cases}q_{\mathrm{conv}} & \text { in } \Gamma_{\mathrm{c}} \times \Upsilon \\ q_{\mathrm{g}} & \text { in } \Gamma_{\mathrm{g}} \times \Upsilon\end{cases}
$$

(b) Convection-radiation constitutive laws. The standard Newton's constitutive law has 
been adopted to evaluate the heat flux due to convection-radiation phenomena between a body and its surrounding environment. This is written as [see e.g. Malvern (1969); Ziegler (1983)] :

$$
q_{\mathrm{conv}}=-h\left(T-T_{\mathrm{env}}\right), \quad \text { in } \Gamma_{\mathrm{c}} \times \Upsilon,
$$

where $h: \Gamma_{\mathrm{c}} \times \Upsilon \rightarrow \mathbb{R}^{+}$is the convection-radiation coefficient (temperature-dependent) and $T_{\text {env }}: \bar{\Omega}_{\text {out }} \times \Upsilon \rightarrow \mathbb{R}^{+}$is the environmental temperature (defined outside $\Omega$ ).

When a gap appears between bodies (1) and (2), convection-radiation effects are governed by the following constitutive law of the medium separating both bodies [see e.g. Malvern (1969); Ziegler (1983)] :

$$
\begin{gathered}
q_{\mathrm{g}_{(1)}}=-h_{\mathrm{g}}\left(T_{(1)}-T_{(2)}\right) \quad \text { in } \Gamma_{\mathrm{g}_{(1)}} \times \Upsilon, \\
q_{\mathrm{g}_{(2)}}=-q_{\mathrm{g}_{(1)}} \text { in } \Gamma_{\mathrm{g}_{(2)}} \times \Upsilon,
\end{gathered}
$$

where $h_{\mathrm{g}}: \Gamma_{\mathrm{g}} \times \Upsilon \rightarrow \mathbb{R}^{+}$is the gap convection-radiation coefficient that, in generally may depend on the normal gap, the normal contact pressure (both will be defined in next section) and the temperatures $T_{(i)}$ existing at the boundaries between the two bodies [see e.g. Celentano (1994)]. Thus, the gap convection-radiation coefficient is expressed as $h_{\mathrm{g}}=\hat{h}_{\mathrm{g}}\left(g_{\mathrm{n}}, p_{\mathrm{n}}, T_{(i)}\right)$ [see e.g. Wriggers et al. (1989); Wriggers and Miehe (1992)].

\subsection{Mechanical contact model}

(a) Basic definitions. In order to define the contact domain, the following conditions are assumed to hold $\bar{\Gamma}_{f} \cup \bar{\Gamma}_{n f}=\bar{\Gamma}_{\sigma}$ and $\Gamma_{f} \cap \Gamma_{n f}=\emptyset$, where $\Gamma_{f} \in \mathbb{R}^{n_{\mathrm{dim}}-1}$ is the part of $\Gamma_{\sigma}$ subject to mechanical contact effects and $\Gamma_{n f} \subset \mathbb{R}^{n_{\mathrm{dim}}-1}$ is the part of $\Gamma_{\sigma}$ free of such phenomena (see Fig. 2). It should be noted that $\Gamma_{f}$ coincides with $\Gamma_{\mathrm{g}}$ [see e.g. Celentano (1994)].

The contact traction vector $\mathbf{t}^{*}$, which has to be taken into account in the boundary equation (5), is given by:

$$
\mathbf{t}^{*}= \begin{cases}\mathbf{t}_{f} & \text { in } \Gamma_{f} \times \Upsilon \\ \mathbf{0} & \text { in } \Gamma_{n f} \times \Upsilon\end{cases}
$$

where $t_{f}$ is the contact vector [see e.g. Celentano (1994)].

(b) Contact (adherence) constitutive law. The contact vector $\mathbf{t}_{f}$ associated with each boundary $\Gamma_{f}$ can be defined as :

$$
\begin{aligned}
& \mathbf{t}_{f_{(1)}}=-p_{\mathrm{n}} \mathbf{n}_{(1)} \quad \text { in } \Gamma_{f_{(1)}} \times \Upsilon, \\
& \mathbf{t}_{f_{(2)}}=-\mathbf{t}_{f_{(1)}} \quad \text { in } \Gamma_{f_{(2)}} \times \Upsilon,
\end{aligned}
$$

$p_{\mathrm{n}}$ being the normal contact pressure [see e.g. Wriggers and Miehe (1992); Wriggers et al. (1989)].

Denoting as $\mathbf{u}_{(i)}$ the displacement vector of configuration $\varphi_{(i)}$ at $\Gamma_{f_{(n)}}$, the definition of the so called gap or penetration $g_{\mathrm{n}}$ is [see e.g. Wriggers and Miehe (1992); Wriggers et al. (1989)],

$$
g_{\mathrm{n}}=\mathbf{n}_{(1)} \cdot\left(\mathbf{u}_{(1)}-\mathbf{u}_{(2)}\right) \text { in } \Gamma_{f_{(i)}} \times \Upsilon \text {, }
$$

where $\mathbf{n}_{(1)}$ is the outward unit normal to body $\mathscr{B}_{(1)}$, which has been chosen here as the reference configuration for the contact problem. This choice is arbitrary, and the reversal 
is also valid but does not give any new insight on the problem [see e.g. Wriggers and Miehe (1992), Wriggers et al. (1989)].

The following secant contact constitutive law is adopted for $p_{\mathrm{n}}$ [see e.g. Celentano (1994); Wriggers and Miehe (1992); Wriggers et al. (1989)] :

$$
p_{\mathrm{n}}=H\left(g_{\mathrm{n}}\right) E_{\mathrm{n}} g_{\mathrm{n}} \text { in } \Gamma_{f} \times \Upsilon,
$$

where $E_{\mathrm{n}}$ is the normal elastic constitutive modulus (also called the normal asperity stiffness [see e.g. Wriggers and Miehe (1992); Wriggers et al. (1989)] which is temperature-dependent in a general case) and, as above, $H$ is the Heaviside function (see Fig. 5). The condition of perfect impenetrability, characterized of ideally polished surfaces in contact, is asymptotically approached as $E_{\mathrm{n}}$ tends to infinity [see e.g. Wriggers and Miehe (1992); Wriggers et al. (1989)].

\section{WEAK FORM AND FINITE ELEMENT FORMULATION}

In order to obtain the weak form of the quasi-static coupled thermomechanical initial boundary value problem described in Section 2, a space of admissible displacement test functions $\mathscr{V}_{u}$ is defined as $\mathscr{V}_{u}=\left\{\boldsymbol{\eta}_{a} \in\left[H^{1}(\Omega)\right]^{n_{\mathrm{dim}}} \mid \boldsymbol{\eta}_{a}=\mathbf{0}\right.$ on $\left.\Gamma_{u}\right\}$ and the corresponding space of admissible temperature test functions $\mathscr{V}_{T}$ is $\mathscr{V}_{T}=\left\{\eta_{\mathrm{a}} \in H^{1}(\Omega) \mid \eta_{\mathrm{a}}=0\right.$ on $\left.\Gamma_{T}\right\}$ where $H^{1}(\Omega)$ is the standard notation for the Hilbert space [see e.g. Simo (1991)]. Accordingly, an admissible displacement solution space ${ }^{t} \mathscr{L}_{u}$ (for a fixed time $t \in \Upsilon$ ) is defined by ${ }^{t} \mathscr{L}_{u}=\left\{\mathbf{u}(X, t) \boxminus\left[H^{1}(\Omega)\right]^{n} \operatorname{dim} \mid \mathbf{u}(X, t)=\overline{\mathbf{u}}(X, t)\right.$ on $\left.\Gamma_{u}\right\}$ while the admissible temperature solution space ${ }^{t} \mathscr{L}_{T}$ is ${ }^{\prime} \mathscr{L}_{T}=\left\{T(X, t) \in H^{1}(\Omega) \mid T(X, t)=\bar{T}(X, t)\right.$ on $\left.\Gamma_{T}\right\}$ [see e.g. Simo (1991)].

Hence, the integral form of the mentioned problem can be formulated as: find a displacement field $\mathbf{u}(X, t)$ and a temperature field $T(X, t)$ which satisfy the local governing equations such that:

$$
\begin{gathered}
\left\langle\nabla \cdot \boldsymbol{\sigma}, \boldsymbol{\eta}_{a}\right\rangle_{\Omega}+\left\langle\rho_{0} \mathbf{B}_{F}, \boldsymbol{\eta}_{a}\right\rangle_{\Omega}-\left\langle\boldsymbol{\sigma} \cdot \mathbf{n}, \boldsymbol{\eta}_{a}\right\rangle_{\Gamma_{\sigma}}+\left\langle\overline{\mathbf{t}}, \boldsymbol{\eta}_{a}\right\rangle_{\Gamma_{\sigma}}+\left\langle\mathbf{t}^{*}, \boldsymbol{\eta}_{a}\right\rangle_{\Gamma_{\sigma}}=0 \quad \forall \boldsymbol{\eta}_{a} \in \mathscr{V}_{u}, \\
-\left\langle\rho_{0} c \dot{T}, \eta_{a}\right\rangle_{\Omega}-\left\langle\rho_{0} L \dot{f}_{\mathrm{pc}}, \boldsymbol{\eta}_{a}\right\rangle_{\Omega}+\left\langle\boldsymbol{\nabla} \cdot(\mathbf{k} \cdot \boldsymbol{\nabla} T), \eta_{a}\right\rangle_{\Omega}+\left\langle\rho_{0} r, \eta_{a}\right\rangle_{\Omega}-\left\langle T \boldsymbol{\beta}: \dot{\varepsilon}, \eta_{a}\right\rangle_{\Omega} \\
+\left\langle r^{\mathrm{p}}, \eta_{a}\right\rangle_{\Omega}-\left\langle\mathbf{n} \cdot \mathbf{k} \cdot \nabla T, \eta_{a}\right\rangle_{\Gamma_{q}}+\left\langle\bar{q}, \eta_{a}\right\rangle_{\Gamma_{q}}+\left\langle q^{*}, \eta_{a}\right\rangle_{\Gamma_{q}}=0 \quad \forall \eta_{a} \in \mathscr{V}_{T},
\end{gathered}
$$

where $\langle., .\rangle_{\Omega},\langle., .\rangle_{\Gamma_{\sigma}}$ and $\langle., .\rangle_{\Gamma_{q}}$ denote the standard $L_{2}$-pairing [see e.g. Simo (1991)] in $\Omega, \Gamma_{\sigma}$ and $\Gamma_{\mathrm{q}}$ respectively. It is important to note that the contribution of all bodies involved in the problem are summed in eqns (56) [see e.g. Celentano (1994)].

The time integration of eqn (56b) is performed via a generalized mid-point rule algorithm [see e.g. Hughes (1987); Simo (1991); Zienkiewicz and Taylor (1989)]. Let $[t, t+\Delta t] \subset \Upsilon(\Delta t>0)$ be a time subinterval. Assuming that algorithmic approximations of the displacement $\mathbf{u}(X): \Omega \rightarrow \mathbb{R}^{n_{\text {dim }}}$ and the temperature ${ }^{t} T(X): \Omega \rightarrow \mathbb{R}^{+}$are known, the objective is to obtain $\mathbf{u}(X), T(X), \dot{\mathbf{u}}(X)$ and $\dot{T}(X)$ at time $t+\Delta t$. To this end, it is necessary to find ${ }^{t+\Delta t} \mathbf{u}(X)$ and ${ }^{t+\Delta t} T(X)$ which verify the local governing equations such that:

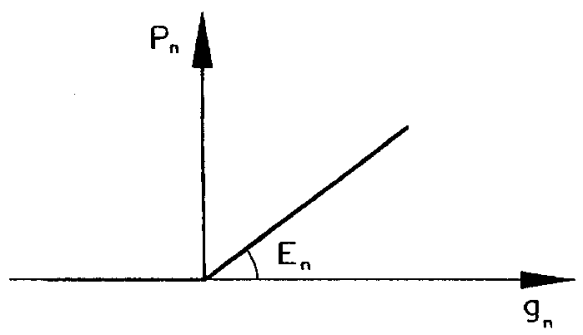

Fig. 5. Normal contact pressure. 


$$
\begin{aligned}
& { }^{t+\alpha \Delta t} \dot{\chi}=\left({ }^{t+\Delta t} \chi-{ }^{t} \chi\right) / \Delta t \\
& { }^{t+\alpha \Delta t} \chi=\alpha^{t+\Delta t} \chi+(1-\alpha)^{t} \chi \quad \text { with } \alpha \in[0,1],
\end{aligned}
$$

$\chi$ being any variable in eqns (56). The option chosen in this work is $\alpha=1$, i.e. backward Euler scheme [see e.g. Hughes (1987); Zienkiewicz and Taylor (1989)].

In the context of the finite element technique [see e.g. Hughes (1987); Zienkiewicz and Taylor (1989)], the discrete problem can be obtained via a spatial Galerkin projection of the semidiscrete problem into finite dimensional subspaces ${ }_{h} \mathscr{V}_{u} \subset \mathscr{V}_{u}$ and ${ }_{h} \mathscr{V}_{T} \subset \mathscr{V}_{T}$ of admissible $C^{0}$ continuous shape functions $\mathbf{N}_{u} \subset \mathscr{C}_{T}$ and $N_{T} \subset \mathscr{V}_{T}$, respectively [see e.g. Simo (1991); Hughes (1987); Zienkiewicz and Taylor (1989)]. Consequently, the admissible "algorithmic" solution spaces ${ }_{h}^{t} \mathscr{L}_{u} \subset{ }^{t} \mathscr{L}_{u}$ and ${ }_{h}^{t} \mathscr{L}_{T} \subset{ }^{t} \mathscr{L}_{T}$ (for fixed time $t \in \Upsilon$ ), also consisting of typical $C^{0}$ functions, are defined such that ${ }_{h}^{t} \mathbf{u}(X) \subset{ }^{t} \mathscr{L}_{u}$ and ${ }_{h}^{t} T(X) \subset{ }^{\prime} \mathscr{L}_{T}$, respectively [see e.g. Hughes (1987); Simo (1991); Zienkiewicz and Taylor (1989)].

Making use of the standard spatial interpolation for the displacement and temperature fields [see e.g. Hughes (1987); Zienkiewicz and Taylor (1989)], it leads to:

$$
\begin{aligned}
& { }_{h}^{t} \mathbf{u}(X)=\mathbf{N}_{u}(X)^{t} U^{(\mathrm{e})} \\
& { }_{h}^{t} T(X)=\mathbf{N}_{T}(X)^{t} T^{(\mathrm{e})}
\end{aligned}
$$

where $\mathbf{N}_{u}=\left[\mathbf{N}_{u_{1}}, \ldots, \mathbf{N}_{u_{n_{n}}}\right] \quad$ with $\quad \mathbf{N}_{u_{i}}=\mathbf{N}_{i} \mathbf{I} \in_{h} \mathscr{V}_{u} \quad$ and $\quad \mathbf{N}_{T}=\left[N_{T_{1}}, \ldots, N_{T_{n_{n}}}\right]$ with $N_{T_{i}}=N_{i} \in{ }_{h} \mathscr{V}_{T}$ both for $i \stackrel{n_{\text {node }}}{=} 1, \ldots, n_{\text {node }}$ and $e=1, \ldots, n_{\text {elem }}$ [see e.g. Hughes (1987); Zienkiewicz and Taylor (1989)].

In above, $\mathbf{N}_{u}$ and $\mathbf{N}_{T}$ are the element shape function matrices for the displacement and temperature interpolation, respectively. Further, ${ }^{t} \mathbf{U}^{(e)}$ is the nodal displacement vector, ${ }^{\prime} \mathbf{T}^{(e)}$ is the nodal temperature vector (the superscript " $\mathrm{e}$ " denotes element values) and $I$ is the identity matrix. It should be noted that the same finite element interpolation is used for each component of $\mathbf{u}$ and $T$ [see e.g. Celentano (1994)]. For simplicity in the notation, the subscript $h$ will be dropped from here onwards.

Following standard procedures [see e.g. Hughes (1987); Zienkiewicz and Taylor (1989)], the global discretized thermomechanical equilibrium equations can be written in matrix form as [see e.g. Celentano (1994)] :

$$
\left\{\begin{array}{l}
{ }^{t+\Delta t} \mathbf{R}_{U}={ }^{t+\Delta t} \mathbf{F}_{U}-{ }^{t+\Delta t} \mathbf{F}_{\sigma}+{ }^{t+\Delta t} \mathbf{F}_{f}=\mathbf{0} \\
\left.{ }^{t+\Delta t} \mathbf{R}_{T}={ }^{t+\Delta t} \mathbf{F}_{T}-\left[{ }^{t+\Delta t} \mathbf{C}-{ }^{t+\Delta t} \mathbf{C}_{p}\right]\right]^{t+\Delta t} \mathbf{T}-{ }^{t+\Delta t} \mathbf{K}^{t+\Delta t} \mathbf{T} \\
-{ }^{t+\Delta t} \dot{\mathbf{L}}_{\mathrm{pc}}-\left[{ }^{t+\Delta t} \mathbf{G}-{ }^{t+\Delta t} \mathbf{G}_{\mathrm{p}}\right]{ }^{t+\Delta t} \mathbf{U}=\mathbf{0}
\end{array}\right.
$$

where $\mathbf{R}_{U}$ and $\mathbf{R}_{T}$ are the mechanical and thermal residual vectors, respectively. The external force vector is $\mathbf{F}_{U}, \mathbf{F}_{\sigma}$ denote the internal force vector and $\mathbf{F}_{f}$ is the mechanical contact vector. Moreover, $\mathbf{F}$ is the external heat flux vector, $\mathbf{C}$ is the capacity matrix, $\mathbf{K}$ is the conductivity matrix and $\dot{\mathbf{L}}_{\mathrm{pc}}$ is the "phase-change" vector rate [see e.g. Celentano et al. (1991); Celentano et al. (1992a,b); Celentano et al. (1993), Celentano et al. (1994), Celentano (1994)]. Furthermore, $\mathbf{G}$ is the thermoelastic coupling matrix, while $\mathbf{C}_{\mathrm{p}}$ and $\mathbf{G}_{\mathrm{p}}$ are coupling matrices due to plastic effects. Once more, ${ }^{t+\Delta t} \dot{\mathbf{U}},{ }^{t+\Delta t} \mathbf{T}$ and ${ }^{t+\Delta t} \dot{\mathbf{L}}_{\mathrm{pc}}$ are computed using eqn (57a).

As usual, all vectors and matrices are assembled from the element contributions in the standard manner [see e.g. Hughes (1987); Zienkiewicz and Taylor (1989)]. The form of the different elemental expressions appearing in eqns (59) can be seen in Box 1, where the superscript $\mathscr{T}$ denotes the transpose symbol and $\mathbf{B}$ is the usual strain-displacement matrix [see e.g. Hughes (1987); Zienkiewicz and Taylor (1989)]. $\mathbf{F}_{c_{U}}$ and $\mathbf{F}_{c_{T}}$ represent the point force vector and the temperature-dependent concentrated heat flux vector respectively, with $n_{c_{V}}$ and $n_{c_{T}}$ being the corresponding number of loaded element nodes. 
Box 1. Element matrices and vectors in the discretized thermomechanical equilibrium equations

$$
\begin{aligned}
& F_{U}^{(e)}=\int_{\Omega^{(c)}} \mathbf{N}_{u}^{\sigma} \mathbf{B}_{\mathrm{F}} \mathrm{d} \boldsymbol{\Omega}+\int_{\Gamma_{a}^{(c)}} \mathbf{N}_{u}^{\sigma} \overline{\mathbf{t}} \mathrm{d} \Gamma_{\sigma}+\sum_{j=1}^{n_{c_{\sigma}}} F_{c_{U}}^{(\mathrm{e})} \\
& F_{o}^{(e)}=\int_{\Omega^{(c)}} \mathbf{B}^{S T} \sigma \mathrm{d} \Omega \\
& F_{f}^{(\mathrm{e})}=\int_{\Gamma_{f}^{(\mathrm{c})}} \mathbf{N}_{u}^{5} \mathrm{t}^{*} \mathrm{~d} \Gamma_{f} \\
& F_{T}^{(\mathrm{e})}=\int_{\Omega^{()^{(j)}}} \mathbf{N}_{T}^{g} \rho_{0} r \mathrm{~d} \boldsymbol{\Omega}+\int_{\Gamma_{\varphi}^{(e)}} \mathbf{N}_{T}^{g} \bar{q} \mathrm{~d} \Gamma_{q}+\int_{\Gamma_{c}^{(e)}} \mathbf{N}_{T}^{g} h T_{\text {env }} \mathrm{d} \Gamma_{c}+\sum_{j=1}^{n_{\tau_{j}}} F_{\tau_{\tau_{j}}}^{(\mathrm{e})} \\
& C^{(c)}=\int_{\mathbf{\Omega}^{(c)}} \mathbf{N}_{T}^{\sigma} \rho_{0} c \mathbf{N}_{T} \mathrm{~d} \Omega
\end{aligned}
$$

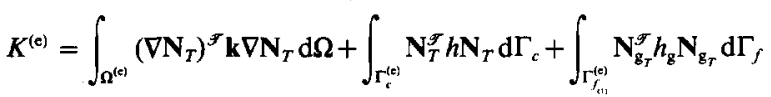

$$
\begin{aligned}
& \dot{L}_{\mathrm{pc}}^{(t)}=\int_{\Omega^{(\mathrm{c})}} \mathbf{N}_{T}^{\mathrm{g}} \rho_{0} L \dot{f}_{\mathrm{pc}} \mathrm{d} \Omega \\
& C_{\mathbf{p}}^{(e)}=\int_{\Omega^{(c)}} \mathbf{N}_{T}^{g} r_{T}^{\mathrm{p}} \mathbf{N}_{T} \mathrm{~d} \Omega \\
& G^{(c)}=\int_{\Omega^{(c)}} N_{T}^{\mathscr{g}} T \beta^{\mathcal{J}} \mathbf{B} \mathrm{d} \Omega \\
& G_{\mathbf{P}}^{(e)}=\int_{\Omega^{(e)}} \mathbf{N}_{T}^{g} \mathbf{r}_{E}^{p / F} \mathbf{B} \mathrm{d} \Omega \\
& \text { with } \\
& \mathbf{N}_{\mathbf{g}_{T}}=\left[\mathbf{N}_{T},-\mathbf{N}_{T}\right]
\end{aligned}
$$

Vector $\dot{\mathrm{L}}_{\mathrm{pc}}$ contains the latent heat effect when ${ }^{t+\Delta t} \dot{f}_{\mathrm{pc}} \neq 0$. Considering that $f_{\mathrm{pc}}$ may present a jump discontinuity for the isothermal problem or a very steep gradient [depending on the size of the phase-change interval $\left.\left(\bar{T}_{1}-\bar{T}_{\mathrm{s}}\right)\right]$ for the non-isothermal one, a nonstandard spatial integration is required to compute $\mathbf{L}_{\mathrm{pc}}$ accurately. The option chosen here is the well-known subdomain integration technique [see e.g. Celentano et al. (1994), Celentano (1994)].

The plastic coupling matrices $\left(\mathbf{C}_{\mathrm{p}}\right.$ and $\left.\mathbf{G}_{\mathrm{p}}\right)$ are zero if no plastic evolutions take place. Similarly, $\mathbf{F}_{f}$ is null when no mechanical contact effects appear [see e.g. Celentano et al. (1993); Celentano (1994)]. It should be noted that the third integral of $\mathbf{K}$ is only evaluated in $\Gamma_{f_{(1)}}$ due to the consideration of matrix $\mathbf{N}_{g_{T}}$ in its expression [see e.g. Celentano (1994)].

Finally, the solution of the non-linear system of eqns (59a) has been attempted by a staggered scheme [see details in the work of Celentano (1994)].

\section{NUMERICAL EXAMPLES}

\subsection{Quenching of a pressurized cylinder}

The quasi-static thermomechanical behaviour of a thick-walled cylinder, initially pressurized with $p_{0}=200 \mathrm{~N} / \mathrm{mm}^{2}$ and subsequently quenched from the initial temperature $T_{0}=320^{\circ} \mathrm{C}$ down to the environmental temperature $T_{\infty}=20^{\circ} \mathrm{C}$, is analysed. This example has been used by several authors as a test problem to study the performance of different solution strategies for thermomechanical analyses and to evaluate the influence of coupling terms [see e.g. Argyris et al. (1981); Armero and Simo (1992a,b)]. Figure 6 describes the problem layout, the geometry and the simple finite element mesh (composed of 20 fournoded axisymmetric elements) used in the computations. Plane strain conditions in the axial direction have been assumed for simplicity (indicated with two continuous lines in Fig. 6). The temperature-dependent mechanical and thermal properties are shown in Tables 1 and 2 , respectively. 


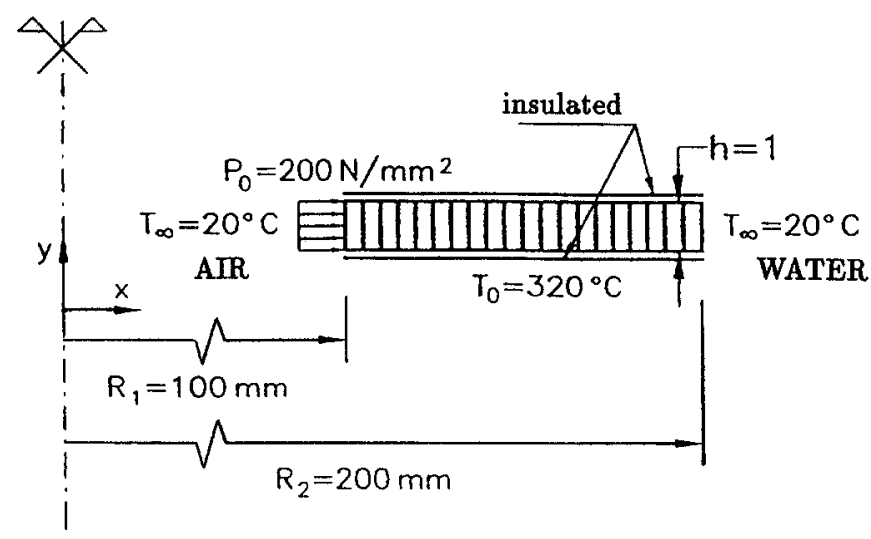

Fig. 6. Quenching of a pressurized cylinder : geometry and finite element mesh.

Four different numerical analyses (using a time step of $10 \mathrm{~s}$ ) have been considered for this problem [see e.g. Celentano (1994)]:

- thermally unidirectional coupled and mechanically elastic (TUC-E),

- bidirectional coupled and mechanically elastic (BC-E),

- thermally unidirectional coupled and mechanically elasto-plastic (TUC-EP),

- bidirectional coupled and mechanically elasto-plastic (BC-EP).

Figures 7 and 8 show the temperature and radial displacement evolutions of a point located at the inside wall. It can be observed that, for the physically realistic material properties, the thermomechanical coupling terms do not play a significant role in the solution considered.

Table 1. Thermal properties

- Density : $\rho_{0}=1.0\left[\frac{\mathrm{kg}}{\mathrm{mm}^{3}}\right]$

- Specific heat capacity: $c^{\mathrm{s}}(T)=1.3 \times 10^{-3}(T-20)+3.50\left[\frac{\mathrm{N} \mathrm{mm}}{\mathrm{kg}{ }^{\circ} \mathrm{C}}\right]$

- Conductivity coefficient : $k(T)=-1.466 \times 10^{-2}(T-20)+45.0\left[\frac{\mathrm{N}}{\mathrm{s}^{\circ} \mathrm{C}}\right]$

- Convection-radiation coefficient (water-solid) $: h_{\text {water }}=1.16\left[\frac{\mathrm{N}}{\mathrm{mm} \mathrm{s}^{\circ} \mathrm{C}}\right]$

- Convection-radiation coefficient (air-solid) $: h_{\text {air }}=0.01\left[\frac{\mathrm{N}}{\mathrm{mm} \mathrm{s}{ }^{\circ} \mathrm{C}}\right]$

- Young's Modulus : $E(T)=-95.0(T-20)+2.2 \times 10^{5}\left[\frac{\mathrm{N}}{\mathrm{mm}^{2}}\right]$

- Poisson's ratio: $v(T)=1.4 \times 10^{-4}(T-20)+0.28$

- Thermal dilatation coefficient : $\alpha_{\mathrm{th}}^{\mathrm{s}}(T)=5.0 \times 10^{-9}(T-20)+12.0 \times 10^{-6}\left[\frac{1}{{ }^{\circ} \mathrm{C}}\right]$

- Thermal hardening function : $\mathscr{G}^{\mathrm{th}}(T)=-0.133(T-20)+320.0\left[\frac{\mathrm{N}}{\mathrm{mm}^{2}}\right]$

- Plastic hardening coefficient : $\mathrm{H}_{\mathscr{6}^{\circ}}=0.0\left[\frac{\mathrm{N}}{\mathrm{mm}^{2}}\right]$ 


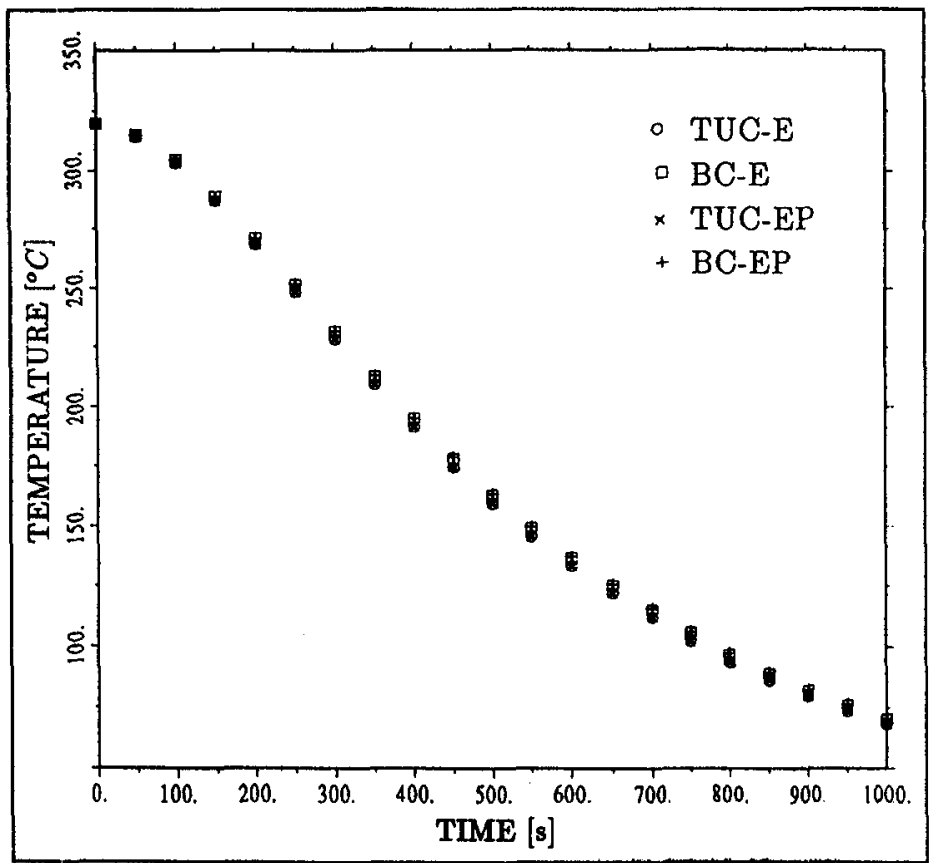

Fig. 7. Quenching of a pressurized cylinder : temperature evolution at the inside wall.

The same analysis has been performed using a physically unrealistic thermal dilatation coefficient $\bar{\alpha}_{\mathrm{th}}^{\mathrm{s}}=5 \alpha_{\mathrm{th}}^{\mathrm{s}}$. The results can be found in Figs 9 and 10. In this situation, the differences between the unidirectional and bidirectional coupled solutions exhibit clearly the importance of the thermomechanical coupling terms.

\subsection{Solidification test}

The cylindrical casting of Nishida et al. (1986) has been analysed. The experiment consisted of casting commercial purity aluminium into an instrumented steel mould. The

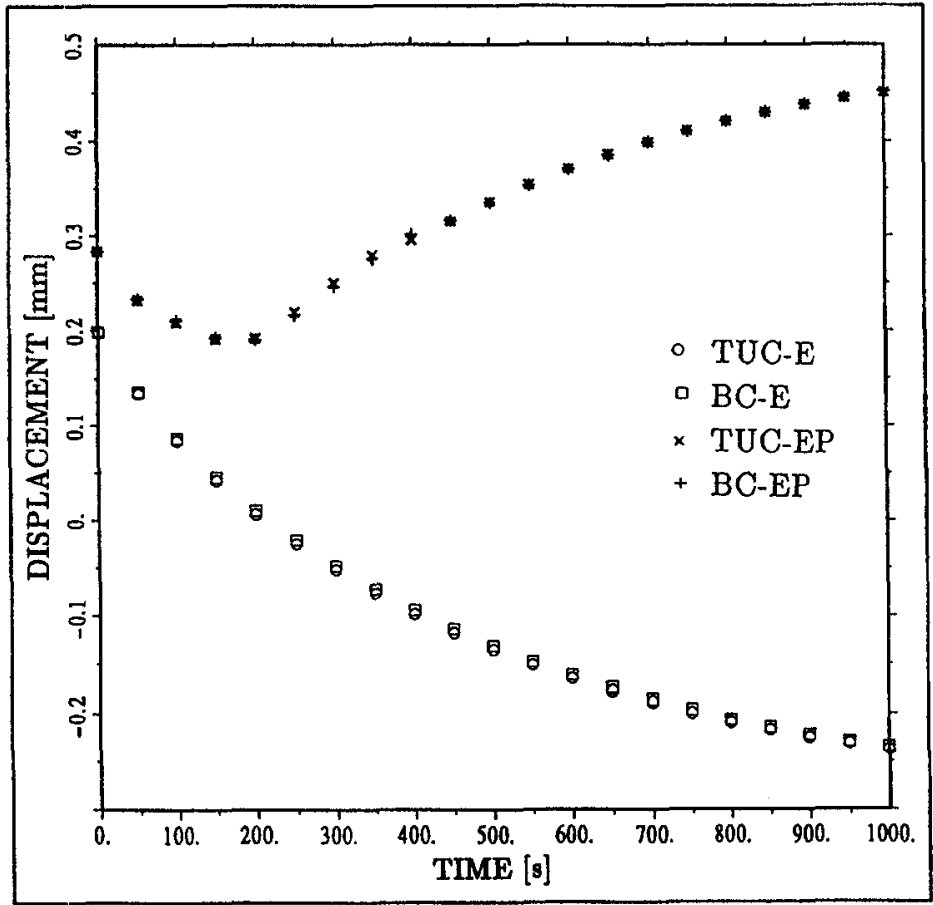

Fig. 8. Queriching of a pressurized cylinder : radial displacement evolution at the inside wall. 


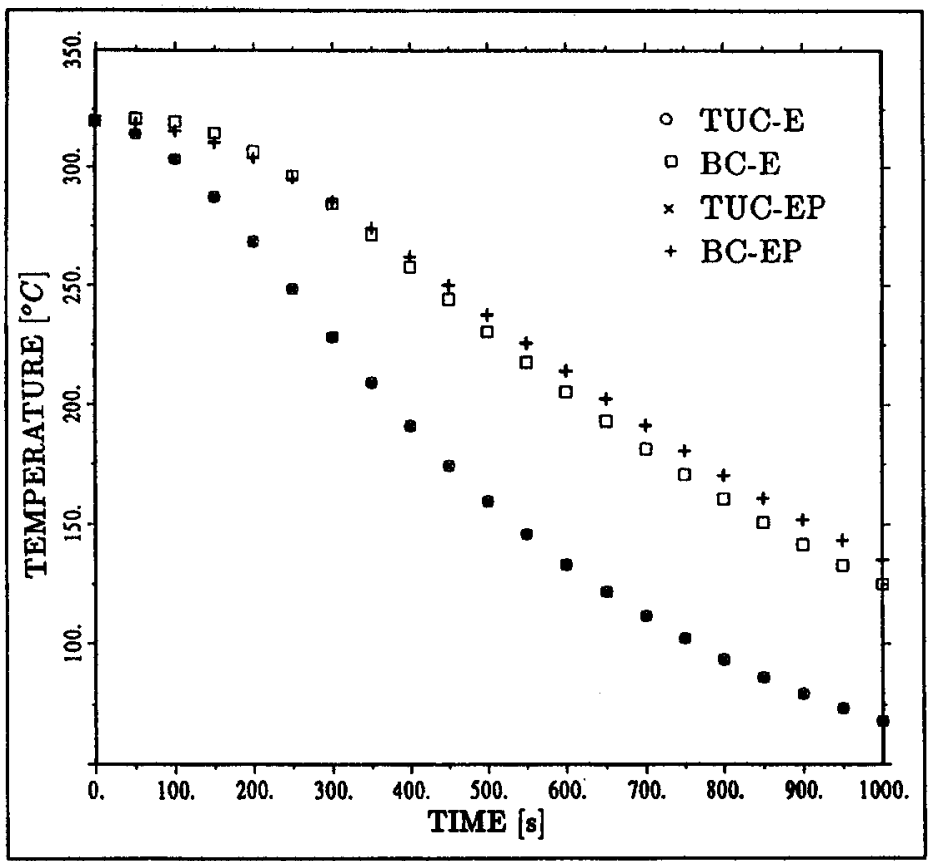

Fig. 9. Quenching of a pressurized cylinder: temperature evolution at the inside wall for $\tilde{\alpha}_{\mathrm{th}}^{\mathrm{s}}=5 \alpha_{\mathrm{th}}^{\mathrm{s}}$.

analysis begins when the mould is completely filled with aluminium in the liquid state, where the initial temperatures are assumed to be $670^{\circ} \mathrm{C}$ for the casting and $200^{\circ} \mathrm{C}$ for the mould. Thermocouples as well as two quartz rods were placed in the mould wall and in the mould cavity [see Fig. 11(a)] in order to measure temperature and radial displacement evolutions, respectively. Geometrical data and thermocouple locations are displayed in Fig. 11(b).

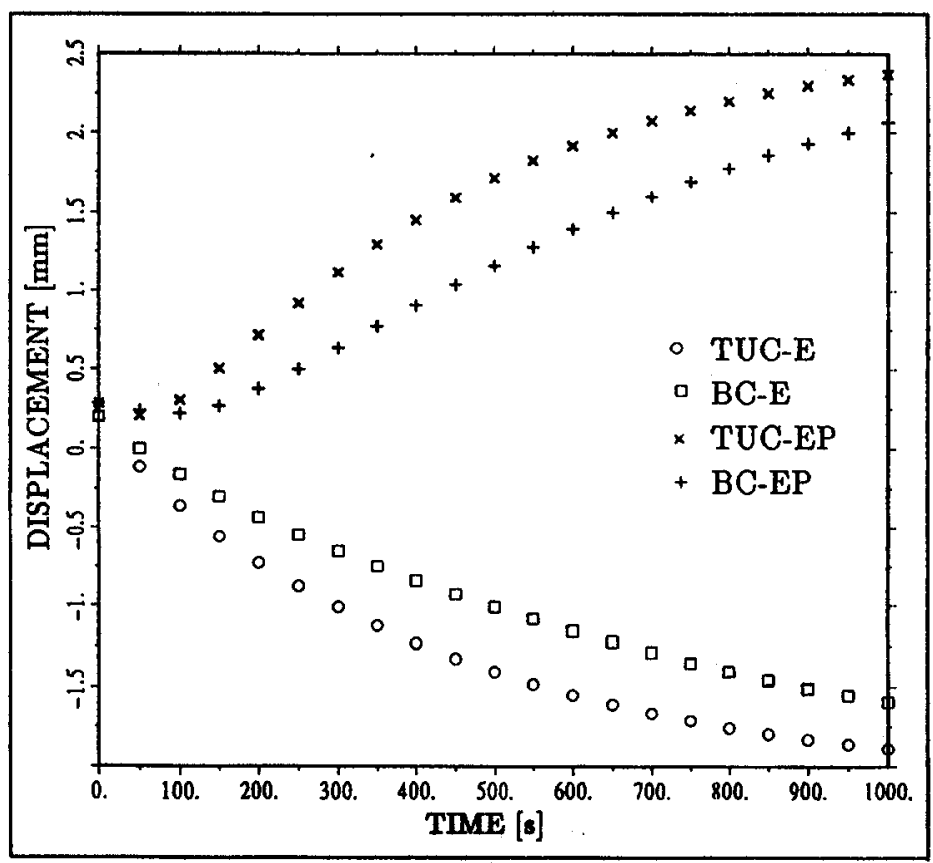

Fig. 10. Quenching of a pressurized cylinder: radial displacement evolution at the inside wall for $\vec{\alpha}_{\mathrm{th}}^{\mathrm{s}}=5 \alpha_{\mathrm{th}}^{\mathrm{s}}$. 

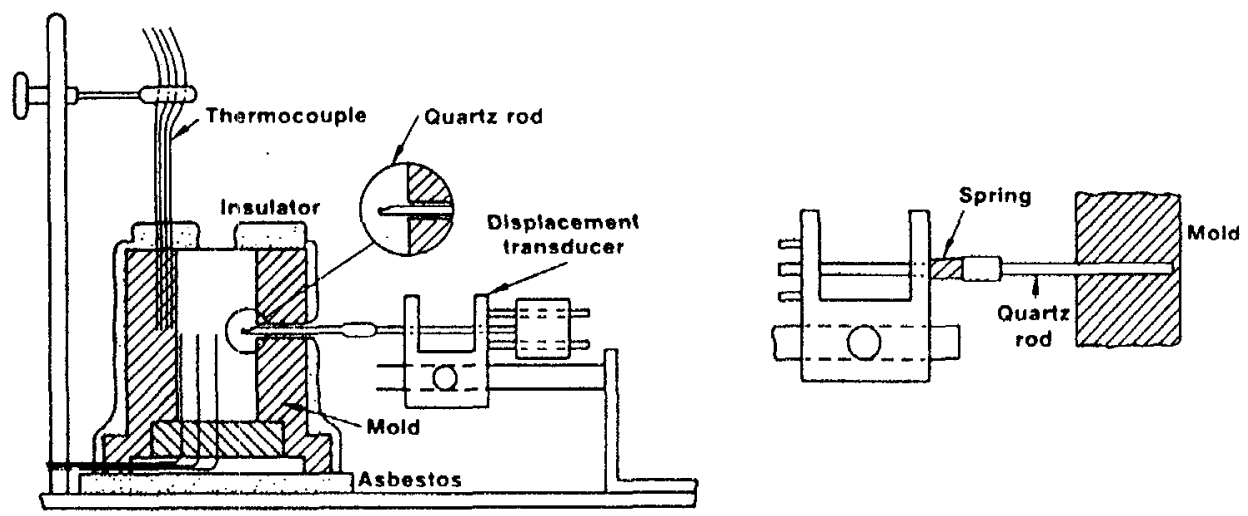

a)
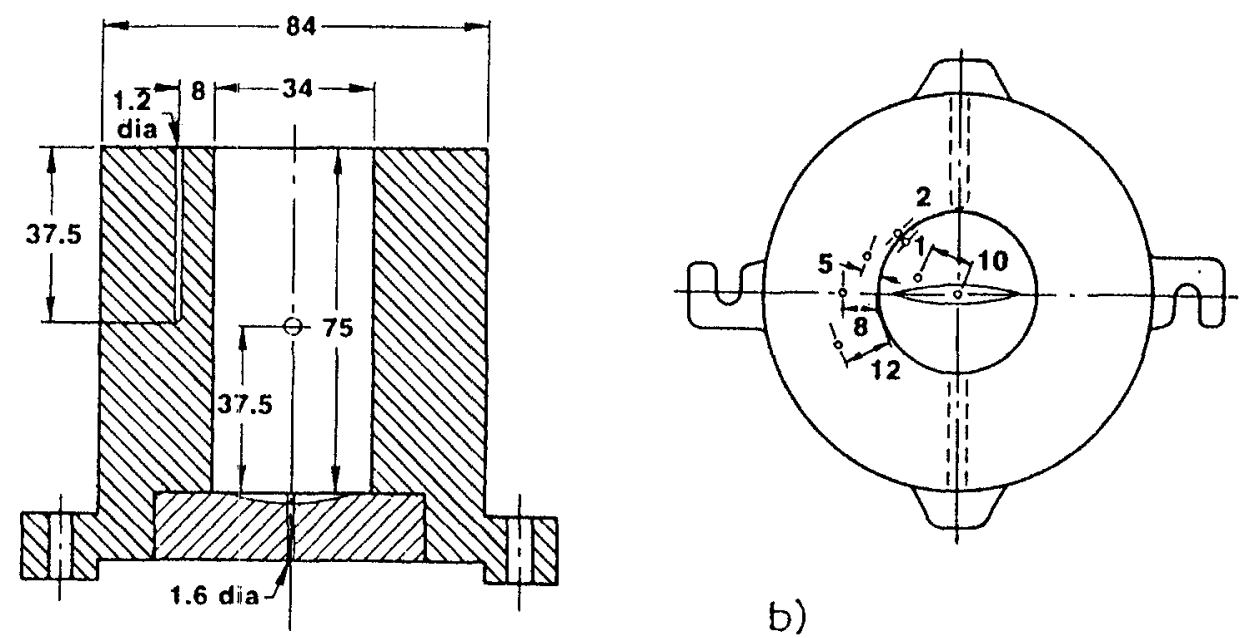

b)

Fig. 11. Solidification test. (a) Schematic of the experimental setup of Nishida et al. (1986) and (b) geometry and thermocouples locations in the mould and casting [drawings from the work of Smelser and Richmond (1988)].

The temperature-dependent thermal and mechanical properties of aluminium are shown in Tables 3 and 4, respectively. It should be noted that for this class of aluminium, only an isothermal liquid-solid phase-change can occur leading, therefore, to $f_{\mathrm{pc}}^{\mathrm{ls}}=H\left(T-\bar{T}_{\mathrm{m}}\right)$ in this case. The steel is assumed to have constant thermal and mechanical properties (see Tables 5 and 6). The convection-radiation coefficient of the metal-mould interface is gap-dependent (see Table 7), while the contact properties between aluminium and steel can be found in Table 8. It should be noted that a frictionless condition has been considered for simplicity. Moreover, the gravity force is the only external force acting on the casting and mould.

The finite element meshes used in the numerical analysis of this problem can be seen in Fig. 12. The first of them [Fig. 12(a)] is a 2D mesh composed of 225 linear triangular axisymmetric elements. Moreover, assuming that all the state variables depend exclusively on the radial direction and time, it is possible to choose a horizontal slice at the midheight of the mould for numerical analysis [see e.g. Celentano (1994); Celentano et al. (1994); Smelser and Richmond (1988)]. This simplificative assumption allows, therefore, the use of a 2D strip [Fig. 12(b)] containing 42 four-noded bilinear axisymmetric elements and a quarter of a 3D strip [Fig. 12(c)] discretized with nearly 600 linear tetrahedral elements. 
- Density: $\rho_{0}=2650.0\left[\frac{\mathrm{kg}}{\mathrm{m}^{3}}\right]$

- Specific heat capacity :

$\begin{array}{cc}c^{s}(T)\left[\frac{\mathrm{kcal}}{\mathrm{kg}{ }^{\circ} \mathrm{C}}\right] & T\left[{ }^{\circ} \mathrm{C}\right] \\ 0.2283 & 100.0 \\ 0.2379 & 200.0 \\ 0.2476 & 300.0 \\ 0.2576 & 400.0 \\ 0.2672 & 500.0 \\ 0.2769 & 600.0\end{array}$

- Conductivity coefficient :

$\begin{array}{cc}k(T)\left[\frac{\mathrm{kcal}}{\mathrm{m} \mathrm{s}^{\circ} \mathrm{C}}\right] & T\left[{ }^{\circ} \mathrm{C}\right] \\ 0.0560 & 100.0 \\ 0.0540 & 200.0 \\ 0.0530 & 400.0 \\ 0.0520 & 600.0 \\ 0.0500 & 659.9 \\ 0.0220 & 660.1 \\ 0.0230 & 800.0\end{array}$

- Melting temperature: $T_{\mathrm{m}}=660.0\left[{ }^{\circ} \mathrm{C}\right]$

- Latent heat : $L=94.44\left[\frac{\mathrm{kcal}}{\mathrm{kg}}\right]$

The variations of $c^{s}(T)$ and $k(T)$ have been assumed to be piecewise linear within the mentioned temperatures.

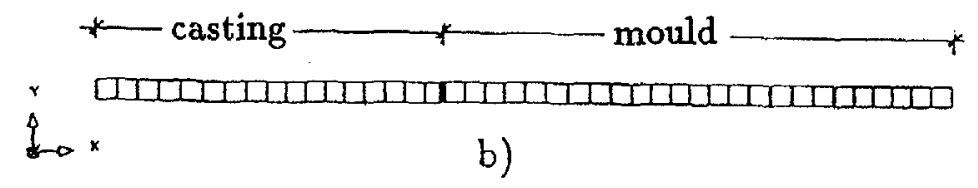

tcastingk mould -
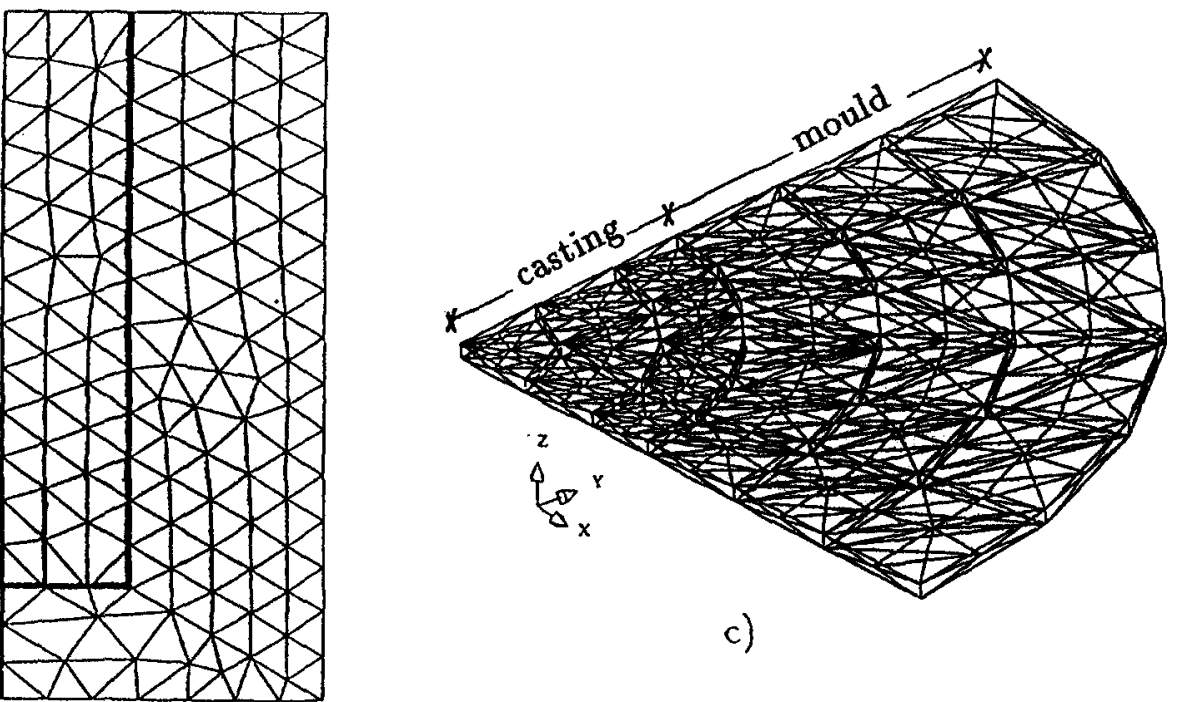

c)

$\varliminf_{0 x}$

a)

Fig. 12. Solidification test : finite element meshes. 
- Young's Modulus :

$\begin{array}{cr}E(T) \times 10^{4}[\mathrm{MPa}] & T\left[{ }^{\circ} \mathrm{C}\right] \\ 6.93 & 25.0 \\ 3.54 & 800.0\end{array}$

- Poisson's ratio : $v=0.37$

- Thermal dilatation coefficient :

$$
\begin{array}{cr}
\alpha_{\mathrm{th}}^{\mathrm{s}}(T) \times 10^{6}\left[\frac{1}{{ }^{\circ} \mathrm{C}}\right] & T\left[{ }^{\circ} \mathrm{C}\right] \\
23.19 & \\
27.86 & 30.0 \\
30.23 & 400.0 \\
30.36 & 659.9 \\
30.12 & 660.1
\end{array}
$$

- Thermal hardening function :

$$
\begin{array}{cc}
\mathscr{C}^{\text {th }}(T)[\mathrm{MPa}] & T\left[{ }^{\circ} \mathrm{C}\right] \\
49.30 & 25.0 \\
0.01 & 660.0
\end{array}
$$

- Plastic hardening coefficient: $H_{\mathscr{C}^{\mathrm{p}}}=0.0[\mathrm{MPa}]$

The variations of $E(T), \alpha_{\mathrm{th}}^{\mathrm{s}}(T)$ and $\mathscr{C}^{\mathrm{th}}(T)$ have been assumed to be piecewise linear within the mentioned temperatures.

Table 5. Thermal properties of steel

- Density : $\rho_{0}=7850.0\left[\frac{\mathrm{kg}}{\mathrm{m}^{3}}\right]$

- Specific heat capacity: $c^{\mathrm{s}}=0.1320\left[\frac{\mathrm{kcal}}{\mathrm{kg}{ }^{\circ} \mathrm{C}}\right]$

- Conductivity coefficient : $k=0.0109\left[\frac{\mathrm{kcal}}{\mathrm{m} \mathrm{s}{ }^{\circ} \mathrm{C}}\right]$

Table 6. Mechanical properties of steel

- Young's Modulus : $E=19.4 \times 10^{4}[\mathrm{MPa}]$

- Poisson's ratio: $v=0.30$

- Thermal dilatation coefficient : $\alpha_{\mathrm{th}}^{\mathrm{s}}=12.0 \times 10^{-6}\left[\frac{1}{{ }^{\circ} \mathrm{C}}\right]$

- Thermal hardening function : $\mathscr{C}^{\text {th }}=210.0[\mathrm{MPa}]$

- Plastic hardening coefficient: $H_{\mathscr{f}^{p}}=0.0[\mathrm{MPa}]$

Table 7. Thermal properties of the castingmould interface

- Convection-radiation coefficient :

\begin{tabular}{cc}
$h\left[\frac{\mathrm{kcal}}{\mathrm{m}^{2} \mathrm{~s}^{\circ} \mathrm{C}}\right]$ & gap $[\mathrm{mm}]$ \\
0.7 & 0.0 \\
0.1 & $>0$ \\
\hline
\end{tabular}

Table 8. Mechanical properties of the casting-mould interface

- Normal asperity stiffness : $E_{\mathrm{n}}=10.0^{10}[\mathrm{MPa} / \mathrm{m}]$ 


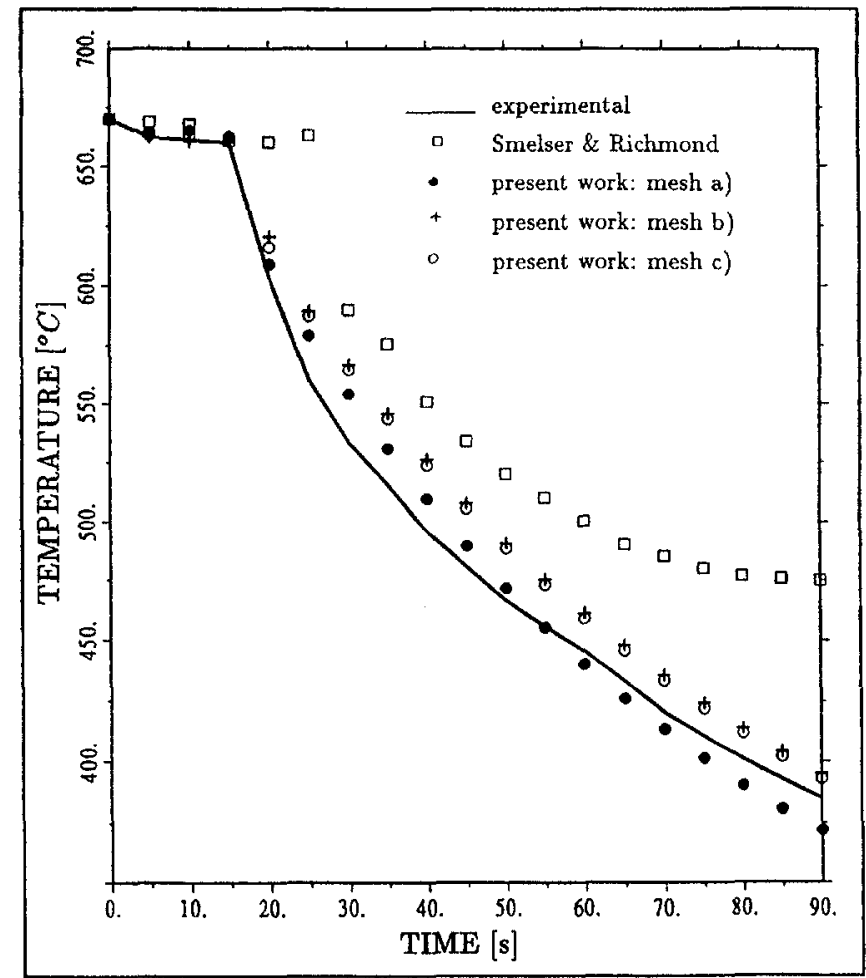

Fig. 13. Solidification test : temperature evolution at the center of the casting.

System (59a) has been solved using the BC numerical strategy with a time step of $5 \mathrm{~s}$. In addition to the features already commented, this fact implies that the energy equation has been solved considering the coupling thermomechanical terms and taking into account the changes in the thermal boundary conditions due to the radial displacements of the specimen.

The temperature evolutions at different points of the mesh are plotted in Figs 13-17. The phase-change effects in the casting can easily be appreciated. Excellent agreement between the numerical results and the experimental ones is obtained.

Figure 18 shows the evolution of the radial displacement at the external aluminium surface. Similarly, Fig. 19 plots the same evolution for a point situated at the inner mould surface. Once more, a good agreement is found between the experimental and numerical results. Further, the progressive change in contact conditions, causing the convectionradiation coefficient to decrease significantly, can clearly be noted in the radial gap evolution depicted in Fig. 20.

The numerical results shown in Figs 13-20 using the three meshes of Fig. 12 show that the simplificative assumption mentioned above seems to be relatively good [fact already noted by Celentano et al. (1994) and Smelser and Richmond (1988)].

Although linear interpolation elements have been used in the analysis [Fig. 12(a) and 12(c)], the well-known volumetric locking effect on the numerical solution [see e.g. Hughes (1980)] for the liquid phase does not occur because the material is assumed to be compressible (the bulk modulus has a finite value) during the whole cooling process. Furthermore, knowing that in the present formulation $\operatorname{tr} \varepsilon^{\mathrm{p}}=0$ ( $\mathrm{tr}$ is the trace symbol), even with $\operatorname{tr} \varepsilon^{\mathrm{e}}=0$ (typical locking when considering a infinite bulk modulus [see e.g. Hughes (1980)], the constraint $\operatorname{tr} \varepsilon=0$ is not satisfied due to the thermal deformations, i.e. $\operatorname{tr} \varepsilon=3 \alpha_{\mathrm{th}}^{\mathrm{s}}$ $\left[T-T_{0}\right]$.

Finally, it is important to emphasize that the usual "numerical trick" of increasing the thermal dilatation coefficient in the liquid phase in order to obtain more realistic displacement evolutions [see e.g. Smelser and Richmond (1988)] is not necessary in this model 


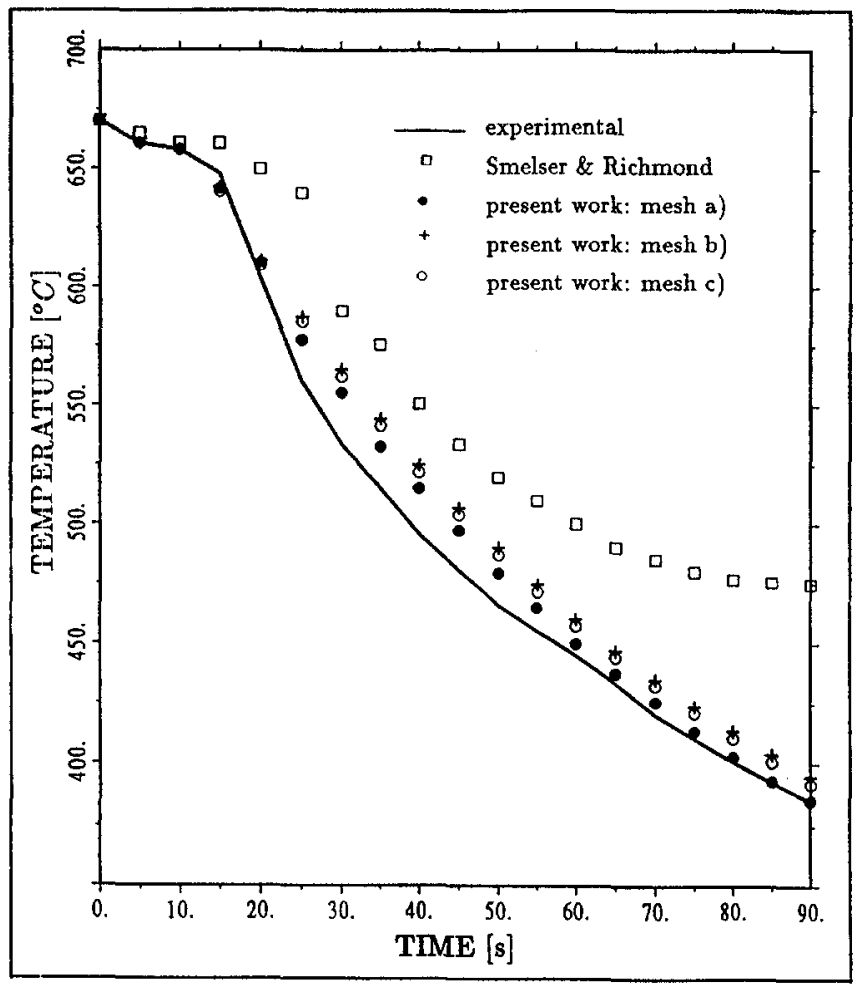

Fig. 14. Solidification test : temperature evolution at a point situated $10 \mathrm{~mm}$ from the center of the casting.

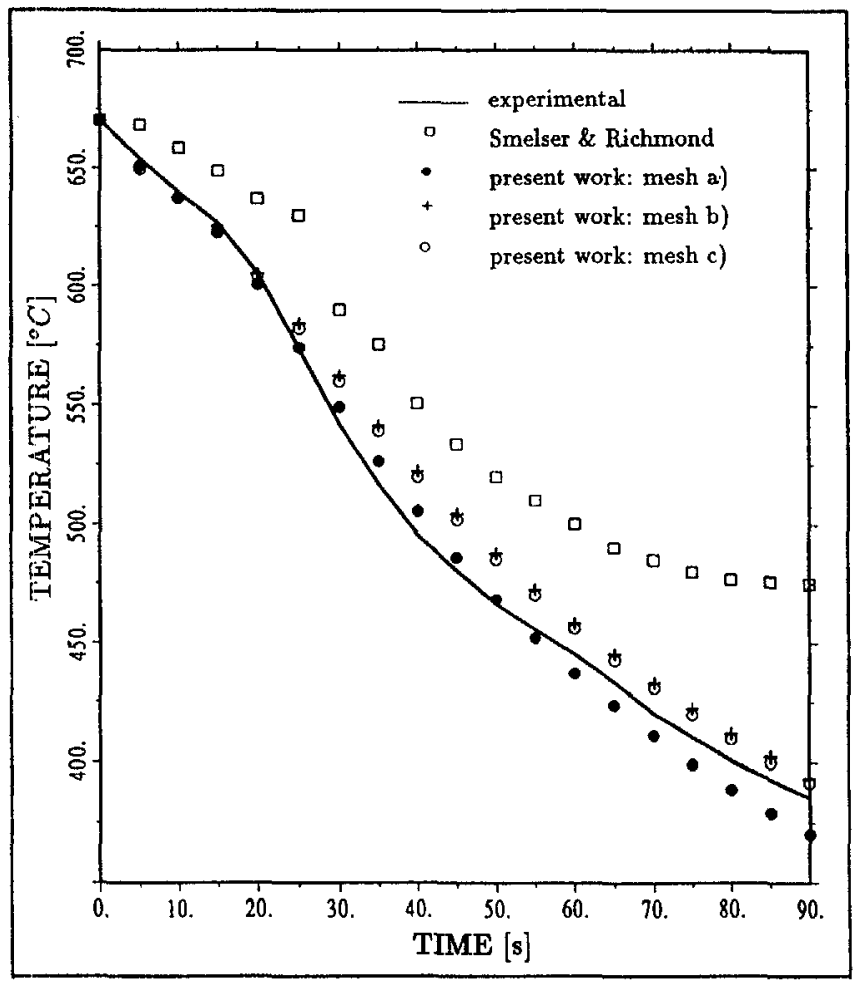

Fig. 15. Solidification test : temperature evolution at the casting surface. 


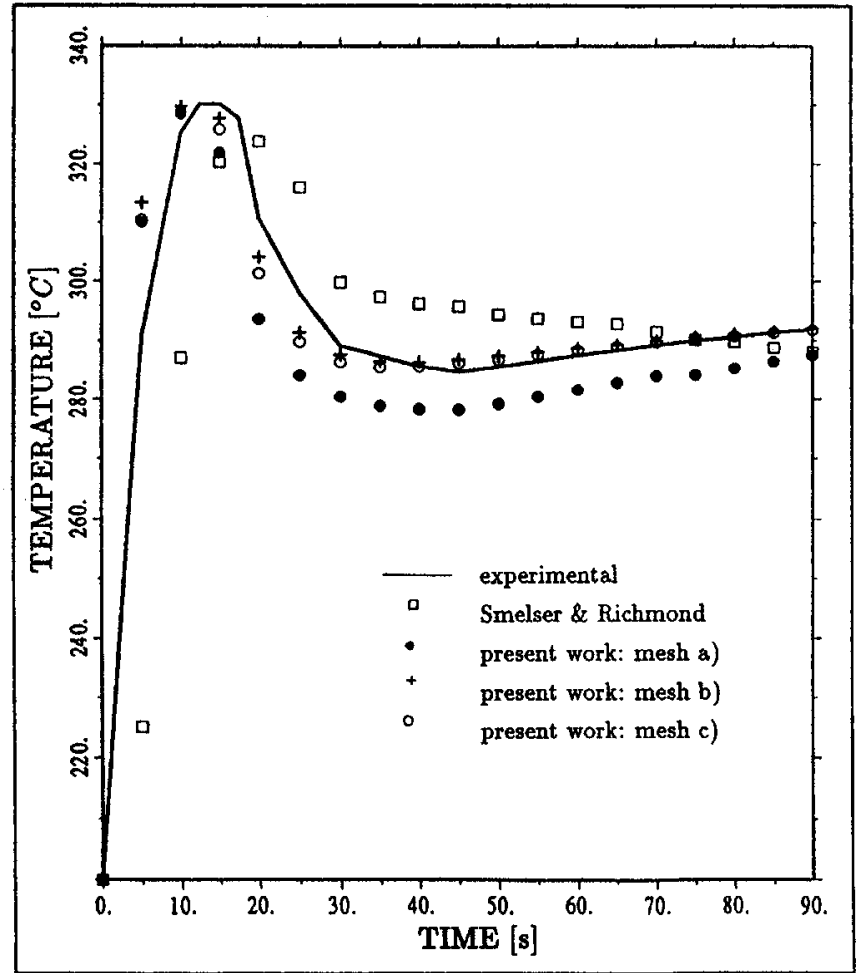

Fig. 16. Solidification test : temperature evolution at the inner mould surface.

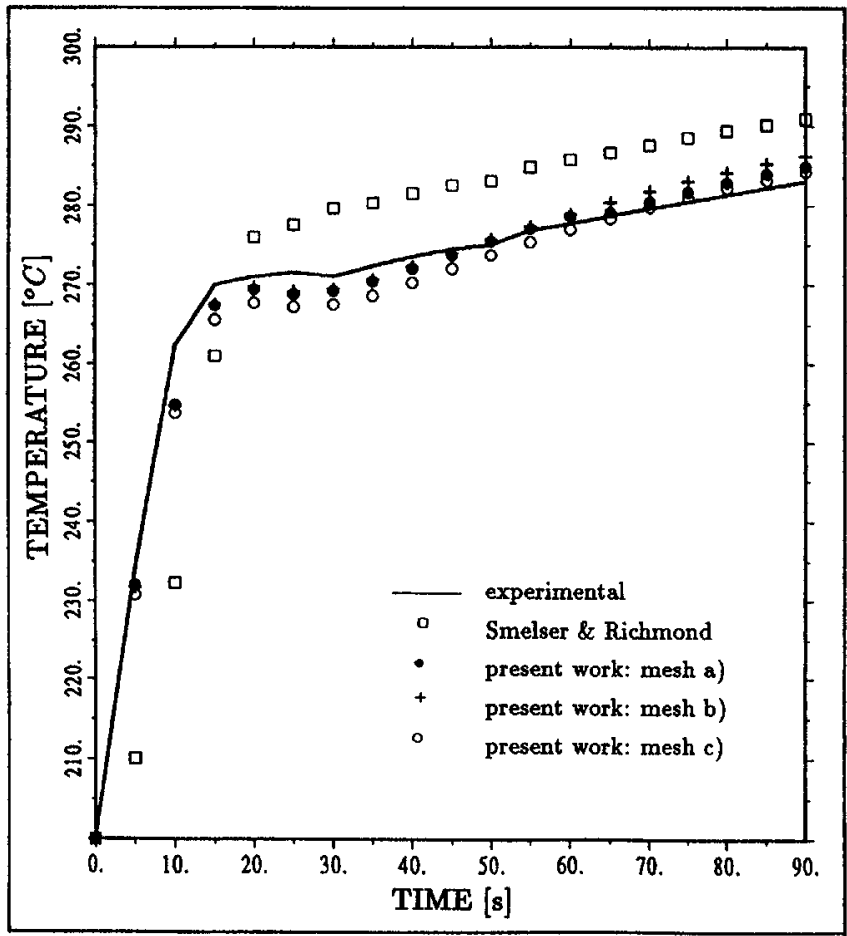

Fig. 17. Solidification test: temperature evolution at a point situated $8 \mathrm{~mm}$ from the inner mould surface. 


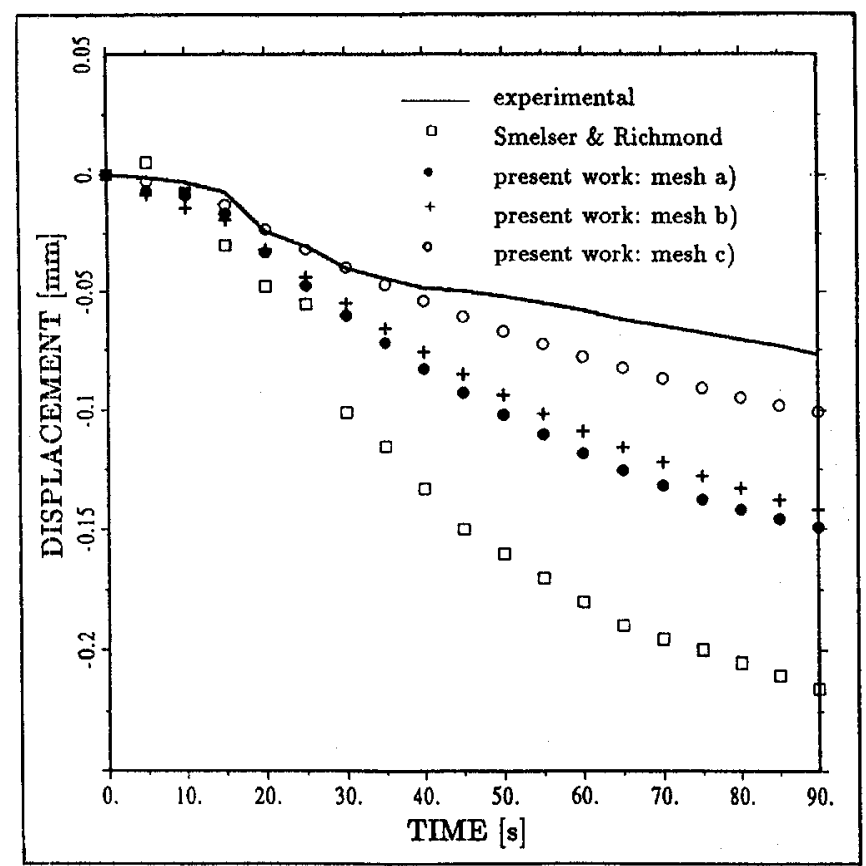

Fig. 18. Solidification test : radial displacement evolution at the casting surface.

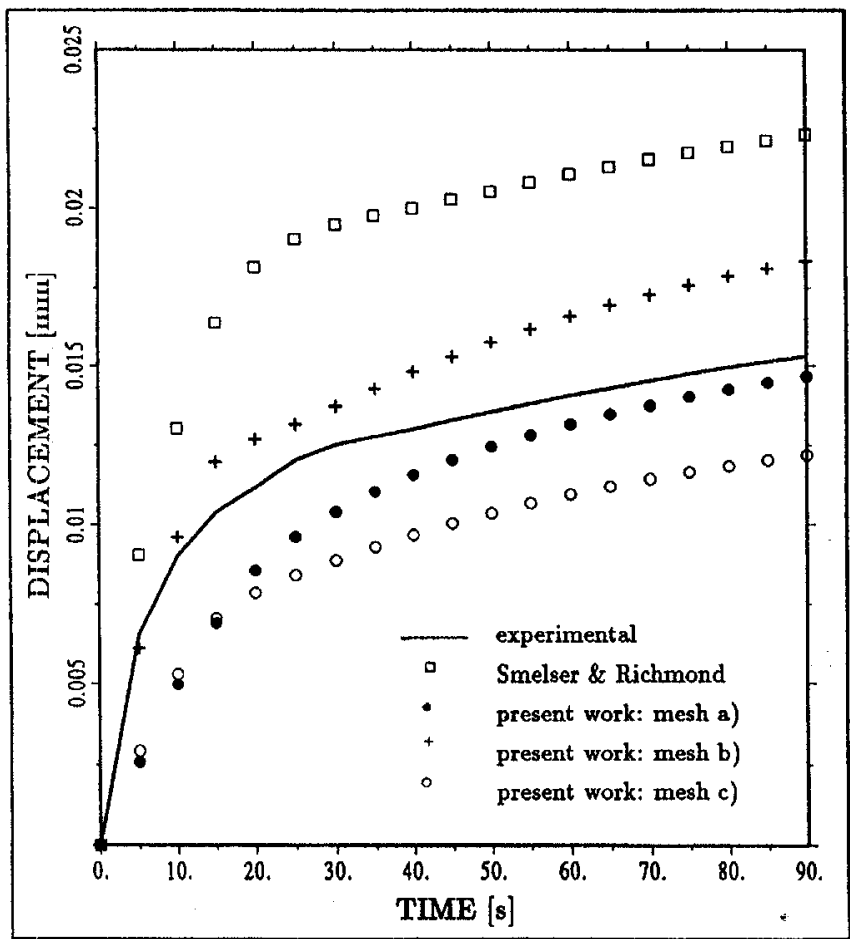

Fig. 19. Solidification test : radial displacement evolution at the inner mould surface.

due to the full volumetric behaviour of the material in this phase given the constitutive equation (42).

\section{CONCLUSIONS}

A thermomechanical model to simulate the solidification problem of casted metals has been presented. The model, based on thermodynamic principles, takes into account different 


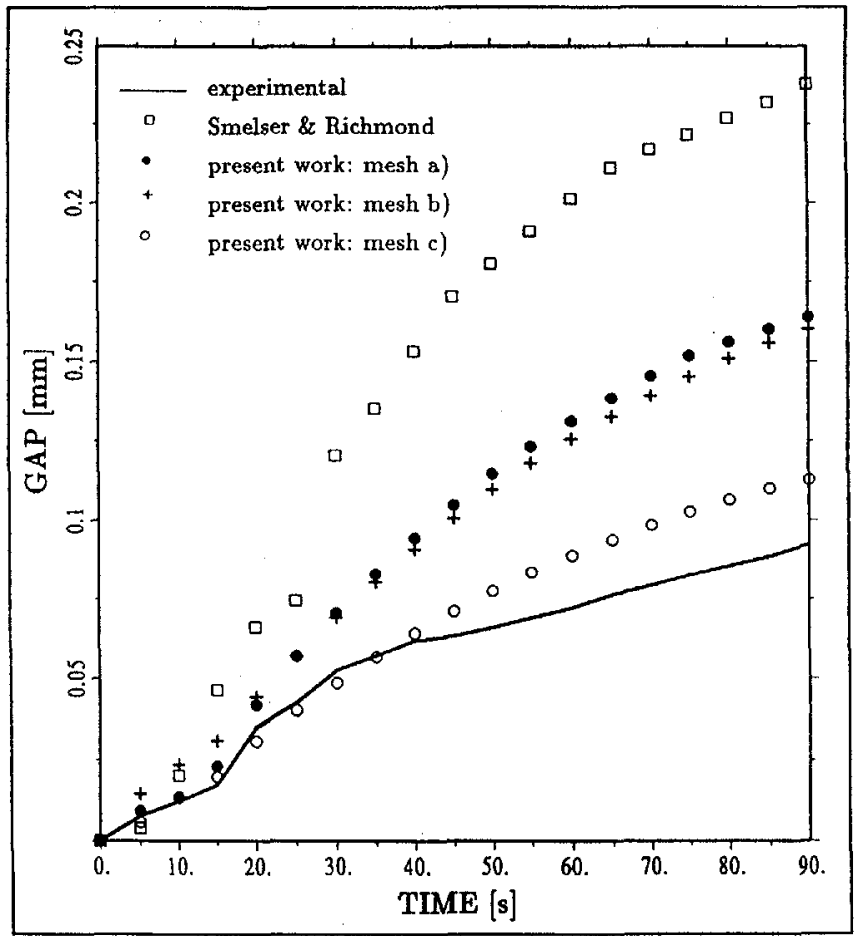

Fig. 20. Solidification test : radial gap evolution.

states of the solidifying material by introducing: a new phase-change function into the definition of the constitutive tensor, some internal plastic variables and a temperaturedependent constitutive law. Besides, full thermomechanical coupling terms and variable mechanical and thermal boundary conditions have been considered.

The model has been implemented in a finite element code. An enhanced staggered scheme has been used in order to solve the highly non-linear fully coupled finite element equations. Finally, the numerical examples analysed show the robustness of the approach.

Further research in this field by the authors includes an extension of the model to account for micro-macro evolution laws, the use of mixing theory to model casting of complex alloys and the implementation of different numerical strategies to enhance even more the cost-effectiveness of the numerical solution.

Acknowledgements-This work has been partially supported by Renault, under contract no. CIMNE/ 10/H5.12.603. The support provided by Brite/Euram Project no. BE-4596 under contract no. BREU-0443 is also gratefully acknowledged.

\section{REFERENCES}

Argyris, J., Vaz, L. and Willam, K. (1981). Integrated finite-element analysis of coupled thermoviscoplastic problems. J. Thermal Stresses 4, 121-153.

Armero, F. and Simo, J. (1992a). Product formula algorithms for nonlinear coupled thermoplasticity : formulation and nonlinear stability analysis. SUDAM Report No. 92-4, Stanford University.

Armero, F. and Simo, J. (1992b). A new unconditionally stable fractional step method for non-linear coupled thermomechanical problems. Int. J. Num. Meth. Engng 35, 737-766.

Bruhns, O. and Služalec, A. (1989). Thermal effects in thermoplastic metal with internal variables. Comput. Struct. 33, 1459-1464.

Cassenti, B. and Annigeri, B. (1989). Thermodynamic constraints on stress rate formulations in constitutive models. Comput. Mech. 4, 429432.

Celentano, D. (1994). A thermomechanical model for solidification problems in metals (in Spanish). Ph.D. Thesis, Universitat Politècnica de Catalunya, Barcelona, Spain.

Celentano, D., Oller, S. and Onate, E. (1991). A constitutive thermomechanical model for solidification of metals. In Journées Numériques de Besançon: Les problemes de changement de phase, pp. 19-30. Held at Arc et Senans, France. 
Celentano, D., Oller, S. and Oñate, E. (1992a) A plastic constitutive model to simulate the solidification in casting problems. In Proceedings of Complas III (Edited by R. Owen, E. Oñate and E. Hinton) pp. 1089-1 102. Pineridge Press/CIMNE, Swansea.

Celentano, D., Oller, S., Oñate, E. and Dardati, P. (1992b). Thermomechanical numerical analysis of an experimental test during solidification. In Proceedings of the "Eurotherm Seminar No. 30: Heat Transfer in PhaseChange Processes", pp. 163-166. Held at Orsay, France.

Celentano, D., Oller, S. and Oñate, E. (1993). A finite element model for thermomechanical analysis in casting problems. In Proceedings of EUROMAT 93, the 3rd European Conference on Advanced Materials and Processes (eds R. Pichoir and P. Costa) pp. 1171-1180. Held at Paris, France.

Celentano, D., Onate. E. and Oller, S. (1994). A temperature-based formulation for finite element analysis of generalized phase-cliange problems. Int. J. Num. Meth. Engng 37, 3441-3465.

Coleman, B. and Gurtin, M. (1967). Thermodynamics with internal state variables. J. Chem. Phys. 47, 597-613.

Hughes, T. (1980). Generalization of selective integration procedures to anisotropic and nonlinear media. In Short Communications, pp. 1413-1418. John Wiley \& Sons, Ltd.

Hughes, T. (1987). The Finite Element Method. Prentice-Hall International, Inc. Englewood Cliffs.

Kleiber, M. (1991). Computational coupled non-associative thermo-plasticity, In Computer Methods in Applied Mechanics and Engineering 90, pp. 943-967. North-Holland.

Lubliner, J. (1972). On the thermodynamics foundations of non-linear solid mechanics. Int. J. Non-linear mech. $7,237-254$

Lubliner, J. (1990). Plasticity Theory. Macmillan Publishing Company, New York.

Malvern, L. (1969). Introduction to the Mechanics of a Continuous Medium. Prentice-Hall, Inc. Englewood Cliffs.

Nishida, Y., Droste, W. and Engler, S. (1986). The air-gap formation process at the casting mold interface and the heat transfer mechanism through the gap. Metall. Trans. B, 17B, 833-844.

Simo, J. (1991). Nonlinear stability of the time-discrete variational problem of evolution in nonlinear heat conduction, plasticity and viscoplasticity. In Computer Methods in Applied Mechanics and Engineering 88, pp. 111-131. North-Holland.

Služalec, A. (1988). An analysis of thermal effects of coupled thermo-plasticity in metal forming processes. Comm. Appl. Num. Meth. 4, 675-685.

Smelser, R. and Richmond, O. (1988). Constitutive model effects on stresses and deformations in a solidifying circular cylinder. In Modeling of Casting and Welding Processes IV, (eds A. F. Giamei and G. J. Abbaschian), The Minerals, Metals \& Materials Soc.

Thévoz, P., Desbiolles, J. and Rappaz, M. (1989). Modelling of equiaxed microstructure formation in casting. Metall. Trans. A, 20A, 311-322.

Williams, J., Lewis, R. and Morgan, K. (1990). An elasto-viscoplastic thermal stress model with applications to the continuous casting of metals. Int. J. Num. Meth. in Engng 30, 579-599.

Wriggers, P. and Miehe, C. (1992). Recent advances in the simulation of thermomechanical contact processes. In Proceedings of Complas III, (Edited by R. Owen, E. Onate and E. Hinton) pp. 325-347. Pineridge Press/CIMNE.

Wriggers, P., Miehe, C., Kleiber, M. and Simo, J. (1989). On the coupled thermo-mechanical treatment of necking problems via finite-element-methods. In Proceedings of Complas II, (Edited by R. Owen, E. Hinton and E. Oñate) pp. 527-542. Pineridge Press/CIMNE.

Ziegler, H. (1983). An Introduction to Thermomechanics. North-Holland Publishing Company.

Zienkiewicz, O. and T'aylor, R. (1989). The Finite Element Method-4th ed., Vols 1 \& 2. McGraw-Hill, London. 